\title{
Spatial variation in stable isotopes and fatty acid trophic markers in albacore tuna (Thunnus alalunga) from the western Indian Ocean
}

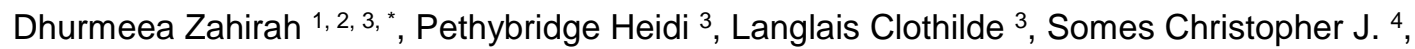 \\ Nikolic Natacha ${ }^{5,6}$, Bourjea Jerome ${ }^{5,6}$, Appadoo Chandani ${ }^{1}$, Bodin Nathalie ${ }^{2,7}$
}

\author{
${ }^{1}$ Department of Biosciences and Ocean Studies, Faculty of Science, University of Mauritius, Réduit, \\ Mauritius \\ ${ }^{2}$ IRD - Research Unit MARine Biodiversity, Exploitation \& Conservation, Victoria, Mahé, Seychelles \\ ${ }^{3}$ CSIRO Oceans and Atmosphere, Hobart, Australia \\ ${ }^{4}$ GEOMAR Helmholtz Centre for Ocean Research Kiel, Düsternbrooker Weg 20, 24105, Kiel, Germany \\ 5 Ifremer, DOI La Réunion, Le Port, Reunion Island, France \\ ${ }^{6}$ IRD, UMR MARBEC, Sainte-Clotilde, Reunion Island, France \\ 7 Seychelles Fishing Authority, Victoria, Mahé, Seychelles
}

*Corresponding author : Zahirah Dhurmeea, email address : $\underline{\text { dzahirah@hotmail.com }}$

\begin{abstract}
:
Albacore tuna (Thunnus alalunga) is a highly economically important species in the western Indian Ocean. However, knowledge of its ecological and nutritional characteristics, essential for proper management of the species, is lacking in the region. The trophodynamics of the Indian Ocean albacore was thus examined using known fatty acid trophic markers (FATMs) of primary producers, nutritional condition indices (NCIs) (omega-3/omega- 6 ratio and total fatty acid content (TFA)), and baseline and lipid corrected stable isotope of carbon ( $\delta 13 \mathrm{Ccorr})$ and nitrogen ( $\delta 15 \mathrm{Ncorr})$, measured in the muscle tissue. We applied generalized additive mixed models to understand the spatiotemporal patterns and drivers of these tracers, taking into consideration several intrinsic and extrinsic variables: fish size, fishing position, month, chlorophyll-a and sea surface temperature (SST). Both chlorophyll-a and SST were significant as single explanatory variables for all tracers with SST being the best predictor for docosahexaenoic acid/eicosapentaenoic acid ratio, the omega- 6 protists FATM, omega-3/omega- 6 ratio and $\delta 15 N$ corr. TFA was best predicted by

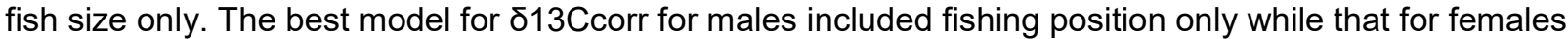
included fish size, fishing position and month. Higher primary productivity, as inferred by high $\delta 13 C$ corr values and diatom contribution, nutritional condition and trophic position, as inferred by high $\delta 15$ Ncorr values, were observed in albacore from the temperate southern waters than in the northern tropical regions. Relationships between environmental variables and corrected stable isotopes, FATMs confirm that ocean warming and changes in primary productivity will impact nutrient flow and energy transfer in the marine food web which may have negative nutritional outcomes for albacore. This knowledge is particularly crucial in areas where oceanographic conditions and seawater temperatures are changing at a fast rate and should also be taken into consideration by fisheries managers.
\end{abstract}




\section{Highlights}

- A multi-tracer approach was taken to examine the trophodynamics of albacore tuna. Albacore inhabiting temperate southern waters feed on a diatom-based food web. Albacore trophic position was lowest in the northern tropical waters. Ontogenetic shifts in stable carbon and nitrogen isotopes were observed. High seawater temperature lowers the nutritional condition of albacore.

Keywords : trophodynamics, phytoplankton, environmental parameters, climate change 
Albacore tuna, Thunnus alalunga (Bonnaterre, 1788), is a temperate and highly migratory species occurring in the tropical, sub-tropical and temperate regions worldwide (Collette and Nauen, 1983). In the western Indian Ocean, albacore is a very important commercial tuna species caught by various types of fishing gears (Coelho et al., 2014; Dhurmeea et al., 2012; Kerwath et al., 2012) and as such is subject to considerable fishing pressure which has resulted in a continuous decline in its biomass over the past years (IOTC, 2019). From an environmental point of view, recent research has shown that the sea surface temperature (SST) in the Indian Ocean is increasing, especially in the western tropical part which has been warming faster than tropical areas in other Oceans (Dong et al., 2014; Du and Xie, 2008) with a rise of $1.2^{\circ} \mathrm{C}$ during the summer period 1901-2012 (Roxy et al., 2014). As the temperature of the ocean increases, picophytoplankton have a tendency to outcompete the larger phytoplankton (Suikkanen et al., 2013). Such size-structured changes are believed to impact the efficiency of energy transfer in marine food webs (Polovina and Woodworth, 2012). The impacts of climate change on the marine community structure, from primary producers to top predators, such as albacore, remain largely unknown but may have socioeconomic implications in terms of catchability and seafood quality (Parrish et al., 2015). Obtaining knowledge of the trophodynamics and nutritional condition of tunas may be an effective means of assessing and predicting the impact that both fisheries and climate can have on marine resources, as well as providing information to support an ecosystem approach to fisheries management (Sinclair et al., 2002).

Trophic tracers such as fatty acids and stable isotopes (SIs) from the muscle tissue of predators are being widely used to obtain important ecological information (Dalsgaard et al., 2003; Revill et al., 2009) due to their ability to integrate feeding histories over an extended time period (months to seasons) (Logan et al., 2008; Madigan et al., 2012). The tissues of marine organisms contain around 20 dominant fatty acids, the relative proportions of which may vary to a large extent within and between populations, species or even individuals of the same species from different regions (Pethybridge et al., 2015a; Sardenne et al., 2016). These variations may stem from a range of abiotic and biotic factors, such as individual size/age, maturity status, tissue, and environment (Dhurmeea et al., 2018; Pethybridge et al., 2015a). Long-chain polyunsaturated fatty acids (PUFAs), especially those of the omega$6(\omega 6)$ and omega-3 ( $\omega 3)$ types, such as eicosapentaenoic acid (EPA) and docosahexaenoic acid (DHA), are known to promote fecundity, egg viability, hatching and fertilization rates, and survival of fish larvae (Furuita et al., 2002; Rainuzzo et al., 1997). The incorporation of fatty acids in the marine food web can only be achieved after synthesis by primary producers, such as algae, diatoms and dinoflagellates which have unique fatty acid signatures (Parrish, 2013). These unique markers can then be traced in higher order consumers to provide insights into predator-prey relationships, food web, energy and nutrient transfer (Dalsgaard et al., 2003; Iverson et al., 2004). For albacore tuna in the south west Pacific Ocean, Parrish et al. (2015) explored the trophic variations using signature fatty acids while Pethybridge et al. (2015a) investigated the spatio-temporal distribution of key fatty acid trophic markers (FATMs) and nutritional condition indices (NCIs) with the aim to identify potential impacts of environmental change on primary producers and higher order consumers. These studies found that projected ocean warming and shifts in primary producer communities, known as tropicalization, is likely to 
adversely affect the supply and availability of essential fatty acids and thus the dietary intake and nutritional condition of albacore, especially in temperate waters.

SI of carbon $\left(\delta^{13} \mathrm{C}\right)$ and nitrogen $\left(\delta^{15} \mathrm{~N}\right)$ vary among organisms as a result of the selective retention of heavier isotopes and excretion of the lighter ones obtained from the diet (Das et al., 2000). Past trophic studies demonstrated that $\delta^{13} \mathrm{C}$ and $\delta^{15} \mathrm{~N}$ values of primary producers are expressed in consumers that inhabit regions longer than their tissue isotopic turnover rates (Lorrain et al., 2015; Olson et al., 2010). $\delta^{15} \mathrm{~N}$ values typically depicts enrichment (3-4\%) from one trophic level to another (Popp et al., 2007) and are useful in the estimation of trophic position (TP) of consumers within a food chain (Post, 2002). On the other hand, $\delta^{13} \mathrm{C}$ values are close to the diet of an organism and are conservative throughout trophic levels with small variations (around 1\%) between diet and consumer (Das et al., 2000). $\delta^{13} \mathrm{C}$ values are useful in distinguishing between the lower or higher-latitude plankton, and pelagic or benthic contribution to food intake (Hobson et al., 1994; Smith et al., 1996). However, the use of SIs to evaluate the trophic dynamics of consumers from various geographic areas may be limited by the variation of seawater isotopic composition which is in turn influenced by the local physical and chemical processes (Vander Zanden and Rasmussen, 1999). This may therefore lead to elevated isotopic variability of primary producers which are at the base of the marine food web. The estimation of TP requires that this variability be taken into account as the natural range of seawater nitrogen isotopes (around 0-12\%) is higher than the increase per TP (Somes et al., 2010). One way to overcome this is by adjusting the SI values of consumers by that of the primary producers such as phytoplankton (Post, 2002). This adjustment has been applied to both $\delta^{15} \mathrm{~N}$ values (Pethybridge et al., 2018; Young et al., 2015) and $\delta^{13} \mathrm{C}$ values (Logan et al., in press) for the examination of large-scale spatial patterns in the trophic structure of marine ecosystems of marine predators.

Whilst the trophic dynamics of albacore have been extensively studied in other regions, little information is available for the Indian Ocean. In the western Indian Ocean, most of the top predator trophodynamics studies undertaken were focused on tropical tunas such as yellowfin (Thunnus albacares), skipjack (Katsuwonus pelamis) and bigeye (Thunnus obesus) tunas, and swordfish (Xiphias gladius) (Ménard et al., 2007; Sardenne et al., 2016; Zudaire et al., 2015). Here, for the first time, using known trophic tracers (SIs, FATMs, NCIs) from previous studies (Parrish et al., 2015; Pethybridge et al., 2015a, 2015b), we examine the trophodynamics of albacore caught in the western Indian Ocean in relation to their biology and environmental variability, and discuss the potential nutritional and ecological implications of a changing climate. More specifically, we use neutral fatty acids (NFAs), which are derived from storage (i.e., neutral) lipids and are subject to only slight modifications from prey to predator, in comparison to structural fatty acids (Robin et al., 2003). Using an ocean circulationbiogeochemistry-isotope model, baseline isotopic variability was adjusted for $\delta^{15} \mathrm{~N}$ and $\delta^{13} \mathrm{C}$ SI values to examine albacore TP and the carbon sources, respectively. As both SIs and fatty acids have their strengths and limitations (El-Sabaawi et al., 2009), combining both trophic tracers produces a more robust picture of the trophodynamics.

\section{Materials and Methods}

\subsection{Field sampling}



the waters of Seychelles (region A) (June-July 2013, April 2014) and Mozambique Channel (region B) (April 2014) were caught by commercial purse-seiners landing in Seychelles and were sampled during processing. Albacore caught by purse-seiners had been stored frozen onboard. All the albacore from Mauritius waters (region C1) were caught by professional artisanal fishermen along the coast of the island using vertical longlines. Samples from region C1 were caught during several periods throughout the year: January-February, May-June and November-December 2014, and January 2015. Albacore in the waters of Reunion Island (region C2) were sampled at sea onboard a commercial longliner chartered in November and December 2013 and from longliners targeting swordfish from May to July 2014. The tuna samples were kept chilled onboard the fishing vessels during the two-week fishing campaign. Albacore from the South African waters (region D) were sampled from November to December 2013 and from April to May 2014. The fresh samples from region D were obtained partly from the catch landed by the commercial pole-and-line fishing boats and at sea by observers. All sampling on land was conducted at processing plants except for those caught by the artisanal fishermen in region C1 which were sampled directly at fish landing sites. Fresh samples were also thus obtained through this artisanal fishery. No ethical approval was required as all fish sampled were dead by sampling time. Samples for NFA analyses excluded samples for region C1 as these were subject to a different fatty acid extraction methodology for another study (Dhurmeea et al., 2018). For each fish, the projected straight distance from the tip of the upper jaw (snout) to the fork of the tail (fork length; $L_{\mathrm{F}}, \mathrm{cm}$ ) and pectoral length $(\mathrm{cm}$; projected straight distance measured with a caliper between the cranial insertion of the pectoral fin and the fork of the tail) were measured. The weight of the viscera, gonads and the total fish weight $(\mathrm{kg})$ were also recorded. Somatic-gutted weight $\left(W_{\mathrm{S}}, \mathrm{kg}\right)$ was calculated as total fish weight $(\mathrm{kg})$ minus visceral and gonadal weights. When $L_{\mathrm{F}}$ was unavailable but pectoral length was known, an estimated $L_{\mathrm{F}}$ was used (i.e., for regions A, C1 and C2) by using length-length linear regressions estimated by Dhurmeea et al. (2016a). Sex of individual fish was determined by macroscopic examination of the gonads. Tissue samples of around $2 \mathrm{~g}$ (wet weight, $w w$ ) were collected from the white muscle (on the dorsal part of the head) and stored frozen at $-80^{\circ} \mathrm{C}$ for subsequent biochemical analyses. Fishing date and position were obtained from the fishing vessels and artisanal fishermen.

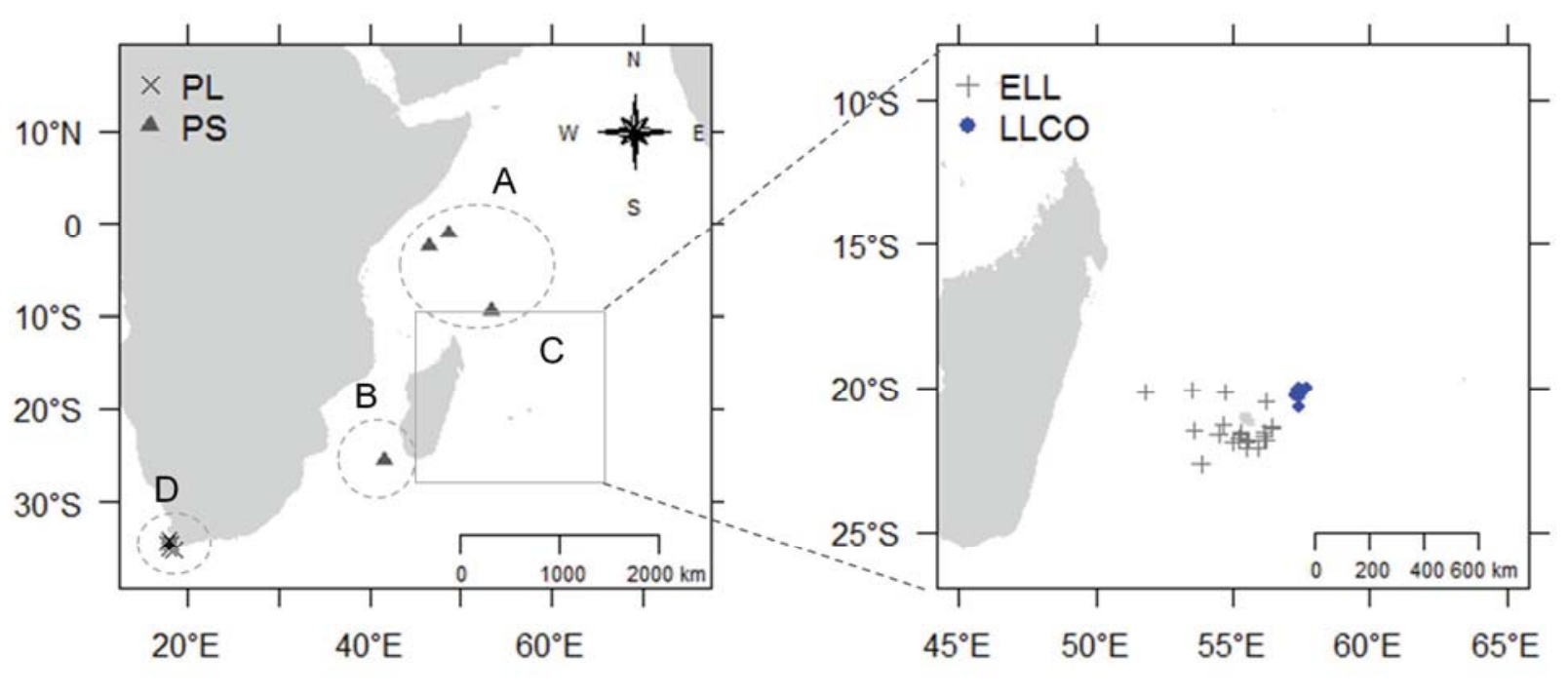


Fig 1. Map of the western Indian Ocean showing positions of sampled albacore tunas by region and gear. Region A: Seychelles waters, region B: Mozambique Channel, region C: Mauritius (C1, blue) and Reunion Island (C2) waters, and region D: South Africa waters. Black crosses: pole-and-liner (PL), grey triangles: purse-seiner (PS), blue dots: coastal fishermen operating in region C1 using longlines (LLCO), grey crosses: longliner (ELL) operating in region C2. Regions are separated by grey dotted lines. (Color to be used in print)

\subsection{Analysis of trophic tracers}

In view of the variation in fatty acids during the reproductive cycle in female albacore (Dhurmeea et al., 2018) and the fact that samples were also collected within the reproductive period and spawning ground of albacore (Dhurmeea et al., 2016b), we tried to limit the effect of reproduction through the analysis of lipids derived from the muscle tissue from male albacore only. However, a larger number of both males and females were examined for SI analysis. Thus, a total of 54 muscle samples from male albacore caught in the regions A, B, C2 and D were analyzed for their NFA content, including FATMs and NCIs. Lipids were extracted in the laboratory in Sète, France, as per Bodin et al. (2009) under high pressure and temperature using dichloromethane. Samples were first freeze-dried and ground using a mixer mill (Retsch MM 200). NFAs were separated using TRACE 1310 gas chromatograph equipped with a FAMEWAXTM column (30 m, $0.32 \mathrm{~mm}$ internal diameter, Restek) and a flameionization detector (FID) (Thermo Scientific), as described in Sardenne et al. (2016). NFAs were expressed as area $\%$ of the total fatty acids in neutral lipids. NFAs that were $<0.8 \%$ in all samples were excluded from the analyses and thus a total of 20 NFAs were analyzed.

The lipid-free samples obtained using the above extraction method were analyzed for $\delta^{13} \mathrm{C}$ and $\delta^{15} \mathrm{~N}$ using a Delta V Advantage isotope ratio mass spectrometer interfaced to a Flash EA 1112 elemental analyzer (Thermo Scientific) following the method by Sardenne et al. (2015). Muscle tissue samples from 313 fish (226 male and 274 female) were analyzed for corrected SIs.

Albacore of variable size range were sampled $\left(L_{\mathrm{F}}: 76-118 \mathrm{~cm}, W_{\mathrm{S}}: 8.43-28.6 \mathrm{~kg}\right.$ for NFA samples and $L_{\mathrm{F}}$ : 74-118 cm, 7.39-28.6 kg for SI samples).

\subsection{Stable isotope analysis and estimation of trophic position}

Phytoplankton $\delta^{15} \mathrm{~N}$ and $\delta^{13} \mathrm{C}$ values were estimated using a model of biogeochemical isotopes (Somes et al., 2017). Baseline corrections from year 2000 of a hindcast simulation include increasing atmospheric $\mathrm{CO}_{2}$ and decreasing $\delta^{13} \mathrm{CO}_{2}$ (i.e. Suess effect). The model comprises a three-dimensional $\left(1.8^{\circ} \times 3.6^{\circ}\right.$ x 19 vertical levels) ocean circulation model forced with fixed monthly climatological winds (Weaver et al., 2001). The biogeochemical component includes 2 nutrients, 2 phytoplankton, 1 zooplankton, sinking detritus, dissolved oxygen and inorganic carbon, alkalinity, and $\delta^{13} \mathrm{C}$ (Somes and Oschlies, 2015). The SI adjustments were then applied by subtracting the modeled phytoplankton $\delta^{13} \mathrm{C}$ and $\delta^{15} \mathrm{~N}$ values from that of the muscle tissue of individual fish, following Pethybridge et al. (2018). Thus, using the equation by Post (2002), TP was estimated as: 


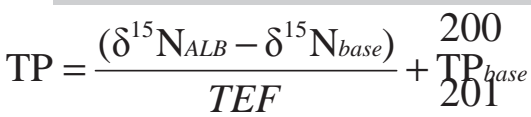

202

203

204

205

206

207

208

209

210

211

212

213

214

215

216

217

218

219

220

221

222

223

224

225

226

227

228

229

230

231

232

233

234

235

236

237

where $\delta^{15} \mathrm{~N}_{A L B}$ is the $\delta^{15} \mathrm{~N}_{\text {corr }}$ of individual fish, $\delta^{15} \mathrm{~N}_{\text {base }}$ is the model predicted phytoplankton value and TEF is the trophic enrichment factor (TEF). We employed a TEF of 2.4\% following methods of Olson et al. (2010) and Lorrain et al. (2015), applied to yellowfin tuna muscle tissue. The baseline trophic position $\left(\mathrm{TP}_{\text {base }}\right)$ was set at 1 as phytoplankton form the base of food webs.

\subsection{Fatty acid trophic markers and nutritional condition indices}

Known FATMs, distinctive of primary producers, were used based on the proportion and ratio of these fatty acids in the tissue of male albacore: detritivorous and grazing primary consumers which include long-chain PUFA protists $(20: 4 \omega 6+22: 5 \omega 6+22: 4 \omega 6)$ (Dalsgaard et al., 2003; Parrish et al., 2012), and the ratio of DHA/EPA which was used to discriminate between a food web based on dinoflagellate (high DHA) versus diatom (high EPA) (Parrish et al., 2015). Both the summed individual fatty acids and DHA/EPA have been shown to be connected to the spatial distribution of fatty acids in albacore tuna (Parrish et al., 2015). The authors also

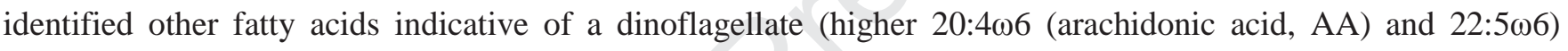

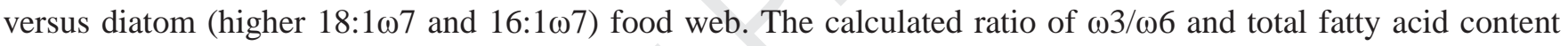
(TFA, \% of tissue $w w$ ) (Parrish et al., 2015; Pethybridge et al., 2015a), which has a positive linear correlation with lipid content (Parrish et al., 2012), were used as nutritional condition indices (NCIs). Larger values of these NCIs indicated better fish nutritional condition (Loef and Walach, 2013).

\subsection{Environmental variables}

Surface environmental variables, available during the sampling years (2014-2015) were selected to assess their impact on trophodynamics of albacore: SST $\left({ }^{\circ} \mathrm{C}\right)$ and surface concentration of chlorophyll- $a$ (Chla, $\mathrm{mg} \mathrm{m}^{-3}$ ). Monthly SST observations were derived from the U.S NOAA National Centre for Environmental information using AVHRR version 2 (Reynolds et al., 2007). Monthly Chla observations were obtained from GlobColour (ACRI-ST, France). Both SST and Chla observations have a spatial resolution of $0.25^{\circ}$ grid and spatial linear interpolation was used to collocate the observations to the sample location.

\subsection{Data analysis}

Based on the minimum length-at-maturity of albacore in the Indian Ocean (Dhurmeea et al., 2016b), fish were classified as either small and immature $\left(W_{\mathrm{G}}<16 \mathrm{~kg}\right.$ and $\left.L_{\mathrm{F}} \leq 94 \mathrm{~cm}\right)$ or large and mature $\left(W_{\mathrm{G}} \geq 16 \mathrm{~kg}\right.$ and/or $L_{\mathrm{F}}>94$ $\mathrm{cm})$. Most small albacore were encountered in region $\mathrm{D}(n=173)$ with a small proportion also being caught in regions A $(n=6)$, B $(n=1)$ and C2 $(n=3)$ for which corrected SIs were analyzed. With regards to the fish for which NFA analysis was made, region D was the only region where small fish were encountered. In addition, in 
the western Indian Ocean, the monsoon circulation largely influences the oceanographic conditions and biological productivity (Schott and McCreary Jr., 2001), which in turn affects the prey of large pelagic predators such as tunas (Vipin et al., 2012). Thus, the following four distinct seasons were taken into consideration in our analyses: the North-Eastern Monsoon (NEM, from mid-November to mid-March), Spring Inter-Monsoon (SIM, from midMarch to mid-May), South-Western Monsoon (SWM, from mid-May to mid-September) and Autumn InterMonsoon (AIM, from mid-September to mid-November). Fish from regions A and B were caught only during SWM and SIM. In regions C1 and C2, fish sampled were captured during AIM, NEM and SWM. In region D, fish were caught in AIM, NEM and SIM.

Using Spearman's ranked correlation, the relationships between NFA and SI trophic tracers were investigated. We also used permutational multivariate analysis of variance (PerMANOVA) to examine the NFA profile of male albacore muscle tissue and to identify potential interactions between region and season, region and $L_{\mathrm{F}}$. Since the NFA data included small fish originating from region D only, analysis of FATMs and NCIs was carried out by regional group (regions $\mathrm{A}, \mathrm{B}, \mathrm{C} 2$, large albacore from region $\mathrm{D}$ and small albacore from region $\mathrm{D}$ ) using analysis of co-variance (ANCOVA). The variations in corrected SI of carbon $\left(\delta^{13} \mathrm{C}_{\text {corr }}\right)$ and nitrogen $\left(\delta^{15} \mathrm{~N}_{\text {corr }}\right)$ were analyzed between the five regions (A, B, C1, C2 and D) using analysis of variance (ANOVA), followed by posthoc Tukey tests. Interactions between region, size class, sex and season were first analyzed prior to the selection of the best ANOVA model for the corrected SIs in the muscle tissue. The results of the interaction between region and season were interpreted carefully because sampling was unbalanced between season and region.

All analyses were conducted using $\mathrm{R}$ version 3.2.2. ( $\mathrm{R}$ Development Core Team, 2017). The vegan package (Oksanen et al., 2018) was used to perform PerMANOVA on the NFAs prior to additional transformation into a distance matrix.

\subsection{Modeling}

Using the gamm4 (Wood and Scheipl, 2017) package in R, we conducted generalized additive mixed models (GAMMs; Wood, 2006) to identify the best predictors for FATMs, NCIs and corrected SI values in the muscle tissue of albacore. Different models incorporating biological $\left(L_{\mathrm{F}}\right)$, geographical position (latitude and longitude) and environmental parameters (SST and Chla) were tested. $L_{\mathrm{F}}$ was included to account for the spatial distribution pattern of different-sized individuals, which is characteristic of albacore tuna. The fishing "sites" within each sampling region, were used as random intercept in the models to account for spatial variation unexplained by the main effects. SST and Chla were separated in the GAMMs because of colinearity $\left(\mathrm{R}^{2}=0.51, P<0.0001\right)$. Time (month) was also included in the models to account for seasonal variation. Different combinations of the variables were then modeled and the best model was identified based on the minimal Akaike Information Criteria (AIC; Burnham and Anderson, 2004) using the AICcmodavg package. The performance of each model was tested using standard diagnostic check on the residuals of the model. Since ANOVA showed that sex had a significant effect on $\delta^{13} \mathrm{C}_{\text {corr }}$ GAMMs for this variable were run for males and females separate. 
All GAMMs and statistical analyses were conducted on arcsine root squared transformed NFAs for normality and homoscedasticity (Underwood, 1997), except for the calculated ratios, corrected SIs and TP which did not necessitate transformation.

\section{Results}

Spearman's ranked correlation revealed highly significant negative correlations between DHA/EPA and both corrected SIs ( $\rho$ values for $\delta^{13} \mathrm{C}_{\text {corr }}=-0.75$ and $\delta^{15} \mathrm{~N}_{\text {corr }}=-0.72$, both $P<0.0001$ ) and NCIs ( $\rho$ values for $\omega 3 / \omega 6=-$ 0.42, $P<0.01$, and TFA $=-0.82, P<0.0001$ ) (Fig 2). Likewise, significant negative correlations were observed between $\omega 6$ protists FATM and both corrected SIs ( $\rho$ values for $\delta^{13} \mathrm{C}_{\text {corr }}=-0.66$ and $\delta^{15} \mathrm{~N}_{\text {corr }}=-0.72$, both $P<$ 0.0001 ) and NCIs ( $\rho$ values for $\omega 3 / \omega 6=-0.67$ and TFA $=-0.74$, both $P<0.0001$ ). However, significant positive correlations were found between NCIs and corrected SI values: $\delta^{13} \mathrm{C}_{\text {corr }}(\rho$ values for $\omega 3 / \omega 6=0.44, P<0.01$, and TFA $=0.68, P<0.0001)$ and $\delta^{15} \mathrm{~N}_{\text {corr }}(\rho$ values for $\omega 3 / \omega 6=0.61$ and TFA $=0.79$, both $P<0.0001)$.

(a)

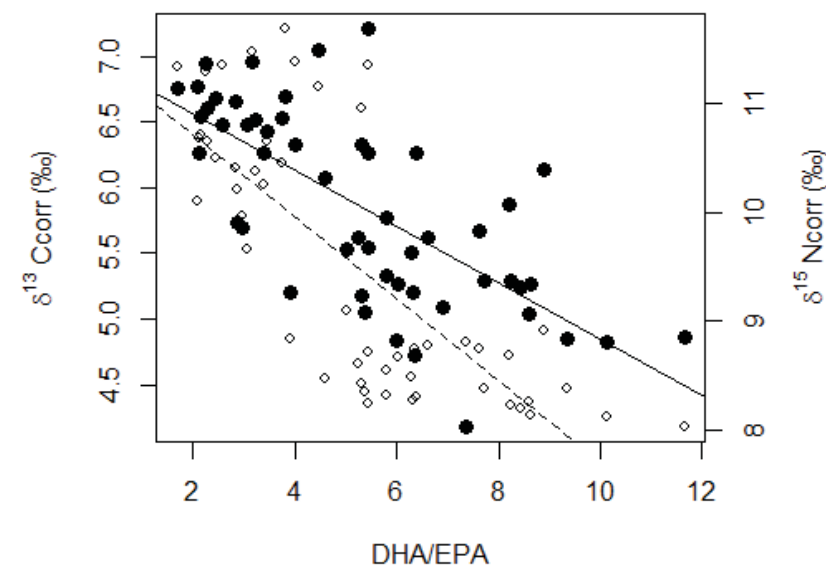

(b)

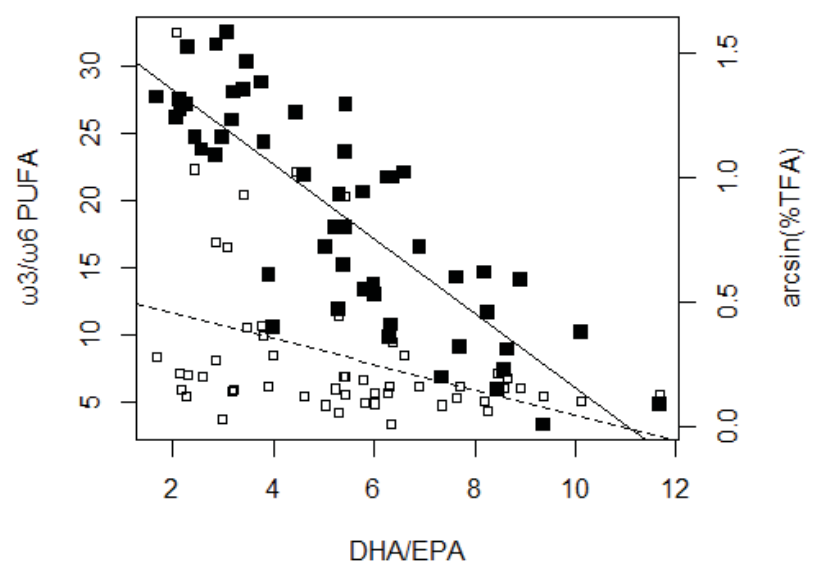

Fig 2. Plots of docosahexaenoic acid/eicosapentaenoic acid (DHA/EPA) with (a) corrected stable isotopes of carbon ( $\delta^{13} \mathrm{C}_{\text {corr }} \%$; white circles) and nitrogen $\left(\delta^{15} \mathrm{~N}_{\text {corr }}\right.$ \% $\%$; dark circles), and (b) nutritional condition indices (omega-3/omega-6 polyunsaturated fatty acid ( $\omega 3 / \omega 6$ PUFA): white squares and arcsine-transformed total fatty acid content (TFA), \% wet weight: dark squares) in the muscle of male albacore tuna from the western Indian Ocean. Dashed and solid lines in each plot represent relationships for white and dark-colored symbols, respectively.

\subsection{Variations of fatty acid profile and fatty acid trophic markers}

A total of 20 NFAs ( $>0.8 \%$ of the total NFAs) from the lipids of muscle tissue of male albacore was analyzed (Table 1). PerMANOVA results showed that the NFA profiles of albacore muscle tissue varied significantly with region, $L_{\mathrm{F}}$ and season, with a slight significant interaction between region and season only (Table 2). Thus, season was used as a covariate when comparing between region and size. SIMPER analysis of dissimilarity revealed that the proportion of DHA was the main contributor to the observed dissimilarity between regions as it is lower in albacore from region D (Table 1). Moreover, ANCOVA identified significant differences in albacore NFA profile 


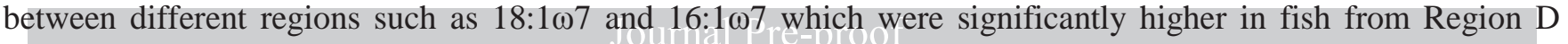
while DHA, AA and 22:5 $\omega 6$ were significantly higher in fish caught in the other regional groups (Table 5.1; all $P$ $<0.05)$

Table 1. Sample sizes, season and mean \pm SD results of biological and neutral fatty acid data (as \% of total fatty acids) for muscle tissue from large and small male albacore tuna collected from different regions from 2013 to 2014 in the western Indian Ocean. Regions A: Seychelles waters, B: Mozambique Channel, C2: Reunion Island, and D: South Africa. $n$ : number of individuals, $L_{\mathrm{F}}$ : fork length $(\mathrm{cm}), W_{\mathrm{S}}$ fish somatic-gutted weight $(\mathrm{kg}), \mathrm{NEM}$ : north-eastern monsoon, SIM: spring inter-monsoon, SWM: south-western monsoon, AIM: autumn intermonsoon, SST: daily sea surface temperature $\left({ }^{\circ} \mathrm{C}\right)$, Chla: daily chlorophyll-a $\left(\mathrm{mg} \mathrm{m}^{-3}\right)$, EPA: eicosapentaenoic acid; DHA: docosahexaenoic acid; AA: arachidonic acid, $\omega$ : omega, SFA: saturated fatty acids, MUFA: monounsaturated fatty acid, PUFA: polyunsaturated fatty acid, LC: long-chain, TFA: total fatty acids (\% weight).

\begin{tabular}{|c|c|c|c|c|c|}
\hline \multicolumn{2}{|l|}{ Fish size } & \multicolumn{2}{|c|}{ Large } & \multirow{2}{*}{ D } & \multirow{2}{*}{$\begin{array}{l}\text { Small } \\
\text { D } \\
\end{array}$} \\
\hline Region & $\mathbf{A}$ & B & $\mathrm{C} 2$ & & \\
\hline$n$ & 8 & 5 & 19 & 7 & 15 \\
\hline$L_{\mathrm{F}}$ & $100.9 \pm 2.2$ & $96.0 \pm 4.6$ & $106.3 \pm 3.5$ & $106.9 \pm 6.4$ & $84.8 \pm 5.3$ \\
\hline$W_{\mathrm{S}}$ & $23.9 \pm 1.2$ & $20.6 \pm 4.5$ & $24.6 \pm 2.3$ & $23.1 \pm 3.2$ & $11.1 \pm 1.8$ \\
\hline Year & 2014 & 2014 & 2013 to 2014 & 2014 & 2013 to 2014 \\
\hline Season & SIM & SIM & $\begin{array}{l}\text { AIM, NEM, } \\
\text { SWM }\end{array}$ & SIM & $\begin{array}{l}\text { AIM, NEM, } \\
\text { SIM }\end{array}$ \\
\hline Latitude $\left({ }^{\circ} \mathrm{E}\right)$ & -9.5 & -25.6 & -22.1 to -21.3 & -34.4 to -34.0 & -35.2 to -34.0 \\
\hline Longitude $\left({ }^{\circ} \mathrm{S}\right)$ & 53.2 & 41.6 & 54.5 to 56.4 & 17.8 to 18 & 17.8 to 18.6 \\
\hline SST & $28.8 \pm 0.0$ & $25.4 \pm 0.0$ & $26.3 \pm 0.4$ & $18.6 \pm 1.2$ & $19.6 \pm 0.7$ \\
\hline Chla & $0.1 \pm 0.0$ & $0.1 \pm 0.0$ & $0.1 \pm 0.0$ & $1.2 \pm 0.7$ & $0.6 \pm 0.6$ \\
\hline $14: 0$ & $0.4 \pm 0.3^{\text {DL,DM }}$ & $0.2 \pm 0.2^{\mathrm{DL}, \mathrm{DM}}$ & $0.8 \pm 0.7^{\mathrm{DL}, \mathrm{DM}}$ & $2.6 \pm 1.6^{\mathrm{A}, \mathrm{B}, \mathrm{C}}$ & $2.7 \pm 2.2^{\mathrm{A}, \mathrm{B}, \mathrm{C}}$ \\
\hline $15: 0$ & $0.3 \pm 0.2$ & $0.2 \pm 0.2$ & $0.4 \pm 0.3$ & $0.6 \pm 0.3$ & $0.5 \pm 0.2$ \\
\hline $16: 0$ & $19.4 \pm 13.0^{\mathrm{DL}, \mathrm{DM}}$ & $11.6 \pm 3.3^{\mathrm{DL}, \mathrm{DM}}$ & $21.1 \pm 7.6^{\mathrm{DL}}$ & $33.4 \pm 4.2^{\mathrm{A}, \mathrm{B}, \mathrm{C}}$ & $28.0 \pm 3.9^{\mathrm{A}, \mathrm{B}}$ \\
\hline $17: 0$ & $0.8 \pm 0.4^{\mathrm{C}}$ & $1.2 \pm 0.4$ & $1.4 \pm 0.4^{\mathrm{A}, \mathrm{DL}}$ & $1.0 \pm 0.3^{\mathrm{C}}$ & $1.2 \pm 0.2$ \\
\hline 18:0 & $15.9 \pm 5.4^{\mathrm{C}, \mathrm{DL}, \mathrm{DM}}$ & $19.2 \pm 5.2^{\mathrm{C}, \mathrm{DL}, \mathrm{DM}}$ & $10.5 \pm 3.2^{\mathrm{A}, \mathrm{B}}$ & $8.9 \pm 2.1^{\mathrm{A}, \mathrm{B}}$ & $10.0 \pm 4.1^{\mathrm{A}, \mathrm{B}}$ \\
\hline $20: 0$ & $0.4 \pm 0.2^{\mathrm{DL}}$ & $0.6 \pm 0.2^{\mathrm{DL}}$ & $0.5 \pm 0.2^{\mathrm{DL}}$ & $0.1 \pm 0.1^{\mathrm{A}, \mathrm{B}, \mathrm{C}, \mathrm{DM}}$ & $0.4 \pm 0.2^{\mathrm{DL}}$ \\
\hline $22: 0$ & $0.4 \pm 0.2$ & $0.45 \pm 0.2$ & $0.3 \pm 0.1$ & $0.4 \pm 0.2$ & $0.3 \pm 0.3$ \\
\hline$\sum$ SFA & $38.0 \pm 9.7$ & $33.5 \pm 4.2^{\mathrm{DL}}$ & $35.4 \pm 8.9^{\mathrm{DL}, \mathrm{DM}}$ & $47.2 \pm 5.6^{\mathrm{B}, \mathrm{C}}$ & $43.3 \pm 6.0^{\mathrm{C}}$ \\
\hline $16: 1 \omega 7$ & $0.9 \pm 0.9^{\mathrm{C}, \mathrm{DL}, \mathrm{DM}}$ & $0.4 \pm 0.2^{\mathrm{C}, \mathrm{DL}, \mathrm{DM}}$ & $2.3 \pm 1.1^{\mathrm{A}, \mathrm{B}, \mathrm{DL}, \mathrm{DM}}$ & $4.5 \pm 1.2^{\mathrm{A}, \mathrm{B}, \mathrm{C}}$ & $5.1 \pm 1.7^{\mathrm{A}, \mathrm{B}, \mathrm{C}}$ \\
\hline $17: 1 \omega 7$ & $0.5 \pm 0.2$ & $0.4 \pm 0.2$ & $0.6 \pm 0.2$ & $0.5 \pm 0.2$ & $0.5 \pm 0.1$ \\
\hline $18: 1 \omega 7$ & $1.7 \pm 0.23^{\mathrm{C}, \mathrm{DL}, \mathrm{DM}}$ & $2.1 \pm 0.4^{\mathrm{DL}, \mathrm{DM}}$ & $2.4 \pm 0.4^{\mathrm{A}, \mathrm{DL}, \mathrm{DM}}$ & $3.1 \pm 0.3^{\mathrm{A}, \mathrm{B}, \mathrm{C}, \mathrm{DM}}$ & $4.1 \pm 0.8^{\mathrm{A}, \mathrm{B}, \mathrm{C}, \mathrm{DL}}$ \\
\hline $18: 1 \omega 9$ & $10.6 \pm 2.8^{\mathrm{C}, \mathrm{DL}, \mathrm{DM}}$ & $10.9 \pm 3.2^{\mathrm{C,DL}}$ & $15.7 \pm 2.4^{\mathrm{A}, \mathrm{B}, \mathrm{DL}, \mathrm{DM}}$ & $23.9 \pm 3.2^{\mathrm{A}, \mathrm{B}, \mathrm{C}}$ & $23.3 \pm 3.9^{\mathrm{A}, \mathrm{B}, \mathrm{C}}$ \\
\hline $20: 1 \omega 9$ & $1.2 \pm 0.3^{\mathrm{DM}}$ & $1.8 \pm 0.6^{\mathrm{DL}}$ & $1.9 \pm 0.5^{\mathrm{DL}, \mathrm{DM}}$ & $1.2 \pm 2.0^{\mathrm{B}, \mathrm{C}, \mathrm{DM}}$ & $3.3 \pm 0.7^{\mathrm{A}, \mathrm{C}, \mathrm{DL}}$ \\
\hline $24: 1 \omega 9$ & $1.2 \pm 0.5^{\mathrm{B}}$ & $2.1 \pm 0.6^{\mathrm{A}, \mathrm{C}, \mathrm{DL}}$ & $0.9 \pm 0.4^{\mathrm{B}}$ & $0.6 \pm 0.1^{\mathrm{B}, \mathrm{DM}}$ & $1.4 \pm 0.6^{\mathrm{DL}}$ \\
\hline$\sum$ MUFA & $16.0 \pm 3.3^{\mathrm{C}, \mathrm{DL}, \mathrm{DM}}$ & $17.8 \pm 4.6^{\mathrm{C}, \mathrm{DL}, \mathrm{DM}}$ & $23.8 \pm 3.1^{\mathrm{A}, \mathrm{B}, \mathrm{DL}, \mathrm{DM}}$ & $33.7 \pm 1.9^{\mathrm{A}, \mathrm{B}, \mathrm{C}}$ & $37.7 \pm 4.3^{\mathrm{A}, \mathrm{B}, \mathrm{C}}$ \\
\hline $18: 2 \omega 6$ & $0.9 \pm 0.5^{\mathrm{DL}}$ & $1.0 \pm 0.5^{\mathrm{DL}}$ & $0.8 \pm 0.1^{\mathrm{DL}}$ & $0.3 \pm 0.4^{\mathrm{A}, \mathrm{B}, \mathrm{C}, \mathrm{DM}}$ & $0.8 \pm 0.4^{\mathrm{DL}}$ \\
\hline $18: 4 \omega 3$ & $0.2 \pm 0.1$ & $0.3 \pm 0.2$ & $0.2 \pm 0.1$ & $0.1 \pm 0.2^{\mathrm{DM}}$ & $0.4 \pm 0.3^{\mathrm{DL}}$ \\
\hline $20: 4 \omega 6$ (AA) & $2.8 \pm 0.6^{\mathrm{DL}, \mathrm{DM}}$ & $3.7 \pm 0.9^{\mathrm{C}, \mathrm{DL}, \mathrm{DM}}$ & $2.4 \pm 0.6^{\mathrm{B}, \mathrm{DL}, \mathrm{DM}}$ & $0.4 \pm 0.3^{\mathrm{A}, \mathrm{B}, \mathrm{C}}$ & $0.7 \pm 0.4^{\mathrm{A}, \mathrm{B}, \mathrm{C}}$ \\
\hline $20: 5 \omega 3$ (EPA) & $3.5 \pm 1.0$ & $4.5 \pm 0.7$ & $4.7 \pm 0.9$ & $4.2 \pm 1.6$ & $3.9 \pm 2.0$ \\
\hline $22: 5 \omega 3$ & $1.0 \pm 0.4^{\mathrm{DL}, \mathrm{DM}}$ & $1.0 \pm 0.3^{\mathrm{DL}, \mathrm{DM}}$ & $1.1 \pm 0.5^{\mathrm{DL}, \mathrm{DM}}$ & $0.1 \pm 0.2^{\mathrm{A}, \mathrm{B}, \mathrm{C}}$ & $0.4 \pm 0.4^{\mathrm{A}, \mathrm{B}, \mathrm{C}}$ \\
\hline $22: 5 \omega 6$ & $1.9 \pm 0.7^{\mathrm{DL}, \mathrm{DM}}$ & $2.3 \pm 0.6^{\mathrm{DL}, \mathrm{DM}}$ & $1.7 \pm 0.7^{\mathrm{DL}, \mathrm{DM}}$ & $0.3 \pm 0.2^{\mathrm{A}, \mathrm{B}, \mathrm{C}}$ & $0.2 \pm 0.1^{\mathrm{A}, \mathrm{B}, \mathrm{C}}$ \\
\hline 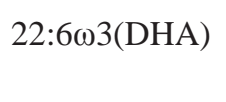 & $32.7 \pm 8.2^{\text {DL,DM }}$ & $32.2 \pm 7.0^{\mathrm{DL}, \mathrm{DM}}$ & $27.8 \pm 7.5^{\mathrm{DL}, \mathrm{DM}}$ & $12.4 \pm 2.9^{\mathrm{A}, \mathrm{B}, \mathrm{C}}$ & $11.0 \pm 4.3^{\mathrm{A}, \mathrm{B}, \mathrm{C}}$ \\
\hline
\end{tabular}




\begin{tabular}{|c|c|c|c|c|c|}
\hline$\sum$ PUFA & $45.9 \pm 11.1^{\mathrm{DL}, \mathrm{DM}}$ & $48.3 \pm 8.4^{\text {DL,DM }}$ & $\operatorname{Pr} 40.8 \pm 9.4^{\mathrm{DL}, \mathrm{DM}}$ & $19.0 \pm 4.9^{\mathrm{A}, \mathrm{B}, \mathrm{C}}$ & $18.9 \pm 7.4^{\mathrm{A}, \mathrm{B}, \mathrm{C}}$ \\
\hline$\omega 3$ & $38.6 \pm 9.5^{\mathrm{DL}, \mathrm{DM}}$ & $39.1 \pm 7.5^{\mathrm{DL}, \mathrm{DM}}$ & $34.7 \pm 8.4^{\mathrm{DL}, \mathrm{DM}}$ & $17.4 \pm 4.3^{\mathrm{A}, \mathrm{B}, \mathrm{C}}$ & $16.4 \pm 6.7^{\mathrm{A}, \mathrm{B}, \mathrm{C}}$ \\
\hline$\omega 6$ & $6.9 \pm 2.1^{\mathrm{DL}, \mathrm{DM}}$ & $8.6 \pm 1.5^{\mathrm{DL}, \mathrm{DM}}$ & $5.8 \pm 1.4^{\mathrm{DL}, \mathrm{DM}}$ & $1.3 \pm 0.9^{\mathrm{A}, \mathrm{B}, \mathrm{C}}$ & $2.1 \pm 1.0^{\mathrm{A}, \mathrm{B}, \mathrm{C}}$ \\
\hline$\omega 3 / \omega 6$ & $5.8 \pm 1.0^{\mathrm{DL}}$ & $4.6 \pm 0.8^{\mathrm{DL}}$ & $6.1 \pm 1.2^{\mathrm{DL}}$ & $18.6 \pm 8.7^{\mathrm{A}, \mathrm{B}, \mathrm{C}, \mathrm{DM}}$ & $9.1 \pm 4.4^{\mathrm{DL}}$ \\
\hline $\mathrm{DHA} \mathrm{EPA}^{\mathrm{FD}}$ & $9.3 \pm 1.3^{\mathrm{B}, \mathrm{C}, \mathrm{DL}, \mathrm{DM}}$ & $7.1 \pm 1.2^{\mathrm{A}, \mathrm{DL}, \mathrm{DM}}$ & $5.9 \pm 1.1^{\mathrm{A}, \mathrm{DL}, \mathrm{DM}}$ & $3.3 \pm 1.3^{\mathrm{A}, \mathrm{B}, \mathrm{C}}$ & $3.1 \pm 0.9^{\mathrm{A}, \mathrm{B}, \mathrm{C}}$ \\
\hline TFA & $1.7 \pm 0.5^{\mathrm{C}, \mathrm{DL}, \mathrm{DM}}$ & $2.9 \pm 0.9^{\mathrm{DL}, \mathrm{DM}}$ & $6.2 \pm 3.1^{\mathrm{A}, \mathrm{DL}, \mathrm{DM}}$ & $15.0 \pm 6.2^{\mathrm{A}, \mathrm{B}, \mathrm{C}}$ & $21.8 \pm 8.4^{\mathrm{A}, \mathrm{B}, \mathrm{C}}$ \\
\hline$\omega 6$ LC-PUFA $^{\mathrm{P}}$ & $5.0 \pm 1.3^{\mathrm{DL}, \mathrm{DM}}$ & $6.3 \pm 1.3^{\mathrm{DL}, \mathrm{DM}}$ & $4.2 \pm 1.2^{\mathrm{DL}, \mathrm{DM}}$ & $0.7 \pm 0.4^{\mathrm{A}, \mathrm{B}, \mathrm{C}}$ & $0.9 \pm 0.6^{\mathrm{A}, \mathrm{B}, \mathrm{C}}$ \\
\hline
\end{tabular}

Superscripts indicate the following:

- Fatty acid trophic markers (FATMs): FD - dinoflagellate versus diatom, $\mathrm{P}$ - $\omega 6$ protists

- Significant differences $(P<0.05)$ between regions: A - different from region A; B - different from region B, C different from region C2; DL and DM - different from large and small albacore from region D, respectively.

Table 2. PerMANOVA results based on Euclidean dissimilarities for testing differences in neutral fatty acid profiles (expressed as a \% of total fatty acids in neutral lipids) of muscle tissue of male albacore tuna caught from 2013 to 2014 in the western Indian Ocean. Factors being tested include region (A: Seychelles, B: Mozambique Channel, C2: Reunion Island, D: South Africa), season (north-eastern monsoon, south-western monsoon, spring inter-monsoon, autumn inter-monsoon), fork length $\left(L_{\mathrm{F}}\right)$ and their interactions when possible.

\begin{tabular}{lcccccc}
\hline Factors & df & SS & MS & Pseudo F & $\mathbf{R}^{2}$ & P value \\
\hline Region & 3 & 0.44 & 0.15 & 26.62 & 0.54 & $\mathbf{0 . 0 0 1}$ \\
$L_{\mathrm{F}}$ & 1 & 0.03 & 0.03 & 5.23 & 0.03 & $\mathbf{0 . 0 0 8}$ \\
Season & 3 & 0.07 & 0.02 & 4.35 & 0.09 & $\mathbf{0 . 0 0 2}$ \\
Region* $L_{\mathrm{F}}$ & 3 & 0.03 & 0.01 & 1.90 & 0.04 & 0.062 \\
Region*Season & 1 & 0.02 & 0.02 & 3.07 & 0.02 & $\mathbf{0 . 0 3 0}$ \\
Residuals & 42 & 0.24 & 0.00 & & 0.28 & \\
Total & 53 & 0.83 & & & 1.00 & \\
\hline
\end{tabular}

df: degrees of freedom; SS: sum of squares, MS: mean sum of squares, Pseudo F (F value by permutation). Significant $P$ values are in bold. $P$ values based on 999 permutations (lowest $P$ value possible 0.001 ).

Significant regional differences were observed for DHA/EPA $\left(\mathrm{F}_{(4,46)}=50.6, P<0.0001\right)$ and $\omega 6$ protists FATM $\left(\mathrm{F}_{(4,46)}=77.6, P<0.0001\right)$ (Table 1) which were lowest in fish from region $\mathrm{D}$, compared to the other regional groups. Significant opposite regional differences were observed in TFA $(1.0-37.6 \% w w)\left(\mathrm{F}_{(4,46)}=43.2, P<\right.$ $0.0001)$ and $\omega 3 / \omega 6\left(\mathrm{~F}_{(4,46)}=15.1, P<0.0001\right)$ whereby higher values were observed in albacore caught from region $\mathrm{D}$, especially in the larger fish for $\omega 3 / \omega 6$.

No significant seasonal variations were found for DHA/EPA, $\omega 3 / \omega 6$ and TFA (all $P>0.05$ ). Seasonal variations were observed for $\omega 6$ protists FATM which had higher values during the seasons NEM $(3.45 \pm 1.68)$ and SIM (3.45 \pm 2.52$)$ compared to AIM $(1.14 \pm 1.79)$.

Apart from the FATMs, we also observed significant variations in the proportions of total saturated fatty acids $\left(\sum \mathrm{SFA}\right)\left(\mathrm{F}_{(4,46)}=5.89, P<0.001\right)$, total monounsaturated fatty acids $\left(\sum \mathrm{MUFA}\right)\left(\mathrm{F}_{(4,46)}=77.0, P<0.0001\right)$ and $\sum$ PUFA $\left(\mathrm{F}_{(4,46)}=33.5, P<0.0001\right)$ (Table 1$)$. Whilst the proportion of $16: 0$ was significantly higher in large albacore from region D compared to the other regional groups, lower proportions of 18:0 occurred in the tissues 
with increasing latitude, from regions D to A. An increasing trend in $\sum$ MUFA was observed with decreasing latitude, from regions A to D, mainly due to variations in the proportion of $18: 1 \omega 9$ and $16: 1 \omega 7$. In contrast, the proportion of $\sum$ PUFA decreased with latitude, mostly in response to reduced proportions of DHA in the tissue.

\subsection{Variations of stable isotopes in albacore tissue}

Albacore $\delta^{13} \mathrm{C}_{\text {corr }}$ and $\delta^{15} \mathrm{~N}_{\text {corr }}$ values varied significantly between region, size and season (Tables 3 and 4 ). Significant interactions were observed between region and size, and sex and season for $\delta^{13} \mathrm{C}_{\text {corr }}$, and between region and size for $\delta^{15} \mathrm{~N}_{\text {corr }}$. In the muscle, $\delta^{13} \mathrm{C}_{\text {corr }}$ varied from 3.39\%o to 7.45\%o (average: $5.11 \pm 1.01 \%$ ) and $\delta^{15} \mathrm{~N}_{\text {corr }}$ values ranged from $7.79 \%$ o to $11.80 \%$ (average: $9.88 \pm 0.88 \%$ ). Modeled phytoplankton $\delta^{13} \mathrm{C}$ values ranged from $-23.8 \%$ to $-21.5 \%$ (average: $-22.57 \pm 0.99 \%$ ) while $\delta^{15} \mathrm{~N}$ values ranged from $2.16 \%$ o to $3.57 \%$ o (average: $2.95 \pm 0.40 \%$ ).

Table 3. Sample sizes, season and mean \pm SD results of biological, environmental, trophic position (TP), carbon and nitrogen stable isotope values for phytoplankton $\left(\delta^{13} \mathrm{C}_{\text {phyt }}\right.$ and $\delta^{15} \mathrm{~N}_{\text {phyt }}, \%$ ), and uncorrected $\left(\delta^{13} \mathrm{C}\right.$ and $\delta^{15} \mathrm{~N}$, $\%$ ) and corrected carbon and nitrogen stable isotope values $\left(\delta^{13} \mathrm{C}_{\text {corr }}\right.$ and $\delta^{15} \mathrm{~N}_{\text {corr }}$ \% $)$ in the muscle tissue of albacore tuna collected from different regions from 2013 to 2015 in the western Indian Ocean. Regions A: Seychelles, B: Mozambique Channel, C1: Mauritius, C2: Reunion Island and D: South Africa. n: number of individuals, $L_{\mathrm{F}}$ : fork length $(\mathrm{cm}), W_{\mathrm{S}}$ fish somatic-gutted weight $(\mathrm{kg}), \mathrm{NEM}$ : north-eastern monsoon, SIM: spring inter-monsoon, SWM: south-western monsoon, AIM: autumn inter-monsoon, SST - daily sea surface temperature $\left({ }^{\circ} \mathrm{C}\right)$; Chla - daily sea surface chlorophyll- $a$ concentrations $\left(\mathrm{mg} \mathrm{m}^{-3}\right)$.

\begin{tabular}{|c|c|c|c|c|c|}
\hline Region & A & B & $\mathrm{C1}$ & $\mathrm{C} 2$ & D \\
\hline$n$ & 127 & 11 & 49 & 114 & 198 \\
\hline$L_{\mathrm{F}}$ & $96.6 \pm 5.1$ & $94.2 \pm 4.1$ & $99.8 \pm 4.2$ & $102.7 \pm 4.4$ & $86.6 \pm 8.0$ \\
\hline$W_{\mathrm{s}}$ & $21.7 \pm 3.3$ & $19.9 \pm 3.6$ & $20.6 \pm 2.1$ & $22.6 \pm 2.7$ & $12.4 \pm 3.9$ \\
\hline Year & 2013 to 2014 & 2014 & 2014 to 2015 & 2013 to 2014 & 2013 to 2014 \\
\hline Season & SIM, SWM & SIM & $\begin{array}{l}\text { AIM, NEM, } \\
\text { SWM }\end{array}$ & $\begin{array}{l}\text { AIM, NEM, } \\
\text { SWM }\end{array}$ & $\begin{array}{l}\text { AIM, NEM, } \\
\text { SIM }\end{array}$ \\
\hline Latitude $\left({ }^{\circ} \mathrm{E}\right)$ & -9.5 to -1.0 & -25.6 & -20.7 to -20.0 & -22.7 to -20.1 & -35.2 to -34.0 \\
\hline $\begin{array}{l}\text { Longitude } \\
\left({ }^{\circ} \mathrm{S}\right)\end{array}$ & 46.4 to 53.2 & 41.6 & 57.3 to 57.7 & 51.8 to 56.4 & 17.8 to 18.6 \\
\hline SST & $27.2 \pm 1.5$ & $26.3 \pm 0.0$ & $26.8 \pm 0.9$ & $26.0 \pm 0.8$ & $18.8 \pm 1.0$ \\
\hline Chla & $0.4 \pm 0.2$ & $0.2 \pm 0.0$ & $0.2 \pm 0.1$ & $0.1 \pm 0.0$ & $1.1 \pm 0.8$ \\
\hline$\delta^{13} C_{\text {phyt }}$ & $-21.6 \pm 0.0$ & $-22.0 \pm 0.0$ & $-21.7 \pm 0.0$ & $-21.9 \pm 0.1$ & $-23.8 \pm 0.0$ \\
\hline$\delta^{15} N_{\text {phyt }}$ & $3.4 \pm 0.1$ & $2.5 \pm 0.0$ & $2.6 \pm 0.1$ & $2.4 \pm 0.2$ & $3.2 \pm 0.1$ \\
\hline$\delta^{13} \mathrm{C}$ & $-17.2 \pm 0.3^{\mathrm{C} 1, \mathrm{C} 2, \mathrm{D}}$ & $-17.5 \pm 0.3$ & $-17.6 \pm 0.3^{\mathrm{A}}$ & $-17.5 \pm 0.4^{\mathrm{A}}$ & $-17.5 \pm 0.4^{\mathrm{A}}$ \\
\hline$\delta^{15} \mathrm{~N}$ & $12.4 \pm 0.4^{\mathrm{B}, \mathrm{C} 2, \mathrm{D}}$ & $11.9 \pm 0.8^{\mathrm{A}, \mathrm{C} 1, \mathrm{D}}$ & $12.5 \pm 0.6^{\mathrm{B}, \mathrm{C} 2, \mathrm{D}}$ & $12.1 \pm 0.6^{\mathrm{A}, \mathrm{C} 1, \mathrm{D}}$ & $13.8 \pm 0.5^{\mathrm{A}, \mathrm{B}, \mathrm{C} 1, \mathrm{C} 2}$ \\
\hline$\delta^{13} C_{\text {corr }}$ & $4.3 \pm 0.2^{\mathrm{C} 2, \mathrm{D}}$ & $4.6 \pm 0.3^{\mathrm{C} 1, \mathrm{D}}$ & $4.2 \pm 0.3^{\mathrm{B}, \mathrm{C} 2, \mathrm{D}}$ & $4.4 \pm 0.3^{\mathrm{A}, \mathrm{C} 1, \mathrm{D}}$ & $6.3 \pm 0.4^{\mathrm{A}, \mathrm{B}, \mathrm{C} 1, \mathrm{C} 2}$ \\
\hline$\delta^{15} N_{\text {corr }}$ & $8.9 \pm 0.5^{\mathrm{C} 1, \mathrm{C} 2, \mathrm{D}}$ & $9.3 \pm 0.8^{\mathrm{C} 1, \mathrm{D}}$ & $9.9 \pm 0.6^{\mathrm{A}, \mathrm{B}, \mathrm{D}}$ & $9.7 \pm 0.6^{\mathrm{A}, \mathrm{D}}$ & $10.6 \pm 0.6^{\mathrm{A}, \mathrm{B}, \mathrm{C} 1, \mathrm{C} 2}$ \\
\hline TP & $4.7 \pm 0.2^{\mathrm{C} 1, \mathrm{C} 2, \mathrm{D}}$ & $4.9 \pm 0.3^{\mathrm{C} 1, \mathrm{D}}$ & $5.1 \pm 0.3^{\mathrm{A}, \mathrm{B}, \mathrm{D}}$ & $5.0 \pm 0.2^{\mathrm{A}, \mathrm{D}}$ & $5.4 \pm 0.2^{\mathrm{A}, \mathrm{B}, \mathrm{C} 1, \mathrm{C} 2}$ \\
\hline
\end{tabular}
from region B, C1: different from region C1, C2: different from region C2. 
Table 4. ANOVA results for testing differences in corrected stable isotopes of carbon $\left(\delta^{13} \mathrm{C}_{\text {corr }}\right)$ and nitrogen $\left(\delta^{15} \mathrm{~N}_{\text {corr }}\right)$ in the muscle tissue of albacore tuna collected from different regions in the western Indian Ocean from 2013 to 2015. Factors being tested include region (A: Seychelles, B: Mozambique Channel, C1: Mauritius, C2: Reunion Island, D: South Africa), season (north-eastern monsoon, south-western monsoon, spring inter-monsoon, autumn inter-monsoon), size class (small or large), sex and their interactions when possible. Significant $P$ values are in bold.

\begin{tabular}{clrrrrr}
\hline Variable & Factors & df & \multicolumn{1}{c}{ SS } & \multicolumn{1}{c}{ MS } & F value & P value \\
\hline $\boldsymbol{\delta}^{\mathbf{1 3}} \mathbf{C}_{\text {corr }}$ & Region & 4 & 438.39 & 109.60 & 956.39 & $<2.2 \mathbf{e}^{-\mathbf{1 6}}$ \\
& Size class & 1 & 1.17 & 1.17 & 10.18 & $\mathbf{0 . 0 0 1 5}$ \\
& Season & 3 & 4.93 & 1.64 & 14.34 & $\mathbf{5 . 8 0 8}$ \\
& Region*Size & & & & & \\
& class & & 1.53 & 0.51 & 4.45 & $\mathbf{0 . 0 0 4 2}$ \\
& Region*Sex & 4 & 3.01 & 0.75 & 6.56 & $\mathbf{3 . 7 9 7}$ \\
& Region*Season & 4 & 2.03 & 0.51 & 4.42 & $\mathbf{0 . 0 0 1 6}$ \\
& Residuals & 480 & 55.01 & 0.11 & & \\
\hline $\boldsymbol{\delta}^{\mathbf{1 5}} \mathbf{N}_{\text {corr }}$ & Region & 4 & 232.75 & 58.19 & 198.23 & $<2.2 \mathbf{e}^{\mathbf{- 1 6}}$ \\
& Size class & 1 & 3.59 & 3.59 & 12.22 & $\mathbf{0 . 0 0 0 5}$ \\
& Season & 3 & 6.07 & 2.02 & 6.89 & $\mathbf{0 . 0 0 0 1}$ \\
& Region*Size & & & & & \\
& class & 3 & 2.84 & 0.95 & 3.23 & $\mathbf{0 . 0 2 2 2}$ \\
& Region*Sex & 4 & 1.66 & 0.41 & 1.41 & 0.2292 \\
& Region*Season & 4 & 1.64 & 0.41 & 1.40 & 0.2328 \\
& Residuals & 480 & 141.11 & 0.29 & & \\
\hline
\end{tabular}

$\delta^{13} \mathrm{C}_{\text {corr }}$ and $\delta^{15} \mathrm{~N}_{\text {corr }}$ values were significantly higher in albacore from region D, compared to regions A, B, C1 and C2 (all $P<0.0001$ ) (Table 3; Fig 5). The mean TP for albacore in the western Indian Ocean was estimated at 5.12 \pm 0.37 and ranged from 4.25 and 5.92. Pairwise tests showed that fish from region $\mathrm{C} 1$ had similar $\delta^{13} \mathrm{C}_{\text {corr }}$ values to region A ( $p=0.08)$, particularly during SWM $(p=1.00)$, but significantly lower values compared to those from regions $\mathrm{B}$, C2, and $\mathrm{D}$ (all $P<0.05$ ). Also, $\delta^{13} \mathrm{C}_{\text {corr }}$ and $\delta^{15} \mathrm{~N}_{\text {corr }}$ values in fish from regions $\mathrm{A}$ and $\mathrm{B}$ were similar (both $P>0.05)$. Although $\delta^{13} \mathrm{C}_{\text {corr }}$ values of fish from regions $\mathrm{A}$ and $\mathrm{C} 2$ were significantly different $(P<0.05)$, they were similar during SWM (p = 0.73). We noted that $\delta^{13} \mathrm{C}_{\text {corr }}$ values in the muscle were significantly lower in NEM than in SIM (mean difference 0.1561, $P<0.001$ ) and SWM (mean difference 0.1901, $P<0.0001$ ). No significant difference in $\delta^{13} \mathrm{C}_{\text {corr }}$ and $\delta^{15} \mathrm{~N}_{\text {corr }}$ values were observed between regions C1 and C2 (all $P>0.05$ ). In general, $\delta^{15} \mathrm{~N}_{\text {corr }}$ values in the muscle were lower during SIM than SWM (mean difference -0.2362, $P<0.01$ ).

Size effects were observed only in region C2 for $\delta^{13} \mathrm{C}_{\text {corr }}$ and in region $\mathrm{D}$ for $\delta^{15} \mathrm{~N}_{\text {corr }}$ with higher values being observed in larger individuals (mean TP: $5.58 \pm 0.20$ ) than smaller ones (mean TP: $5.40 \pm 0.23$ ) (mean difference $\delta^{15} \mathrm{~N}_{\text {corr }}$ : 0.3834, $P<0.05, \delta^{13} \mathrm{C}_{\text {corr }}$ : 0.8469, $P<0.001$ ). Region $\mathrm{C} 2$ was also the only region to show a significant difference between sexes with higher $\delta^{13} \mathrm{C}_{\text {corr }}$ in the muscle of males than in females (mean difference $0.2708, P<$ $0.01)$. 
The results for different model formulations fitted for the different albacore FATMs, NCIs and corrected SIs are given in SI1 Table.

Both Chla and SST were significant as single explanatory variables for the muscle FATMs, NCIs and corrected SIs, and were explained by deviance 60-92\%. In fact, DHA/EPA, $\omega 6$ protists FATM, $\omega 3 / \omega 6$ and $\delta^{15} \mathrm{~N}_{\text {corr }}$ ( sexes combined), were best predicted by the stand-alone model with SST with variability of $78.6 \%, 90.2 \%, 65.2 \%$ and $70.4 \%$, respectively, while $L_{\mathrm{F}}$ only was the best model for TFA with variability of $86.6 \%$. The best model for $\delta^{13} \mathrm{C}_{\text {corr }}$ values in male albacore included the two-dimensional surface of $\mathrm{t}$ (latitude, longitude) alone while the combined model of $L_{\mathrm{F}}$, fishing position and month was the best model for females. Apart from TFA, $L_{\mathrm{F}}$ had clearly a significant effect on $\delta^{13} \mathrm{C}_{\text {corr }}$ and $\delta^{15} \mathrm{~N}_{\text {corr }}$ when both sexes were combined. GAMM using $L_{\mathrm{F}}$ as the only predictor for DHA/EPA, $\omega 6$ protists FATM, $\omega 3 / \omega 6$ and $\delta^{13} \mathrm{C}_{\text {corr }}$ (sexes separate) was not significant (SI1 Table). The stand-alone model with Chla was not identified as the best model for any FATMs, NCIs and corrected SIs although it was significant.

The stand-alone GAMMs with $L_{\mathrm{F}}$ had their intercept identifying a threshold at around $98-100 \mathrm{~cm} L_{\mathrm{F}}$, which were particularly clear for the significant models (Fig 3): the predicted values for TFA decreased linearly (Fig 3) while those for $\delta^{13} \mathrm{C}_{\text {corr }}$ and $\delta^{15} \mathrm{~N}_{\text {corr }}$ showed an overall increase with length (Fig 4). DHA/EPA showed a steep increase with SST with intercept falling at $25^{\circ} \mathrm{C}$. A positive linear trend was observed for the $\omega 6$ protists FATM while TFA exhibited the opposite trend, both having their intercept crossing at $23^{\circ} \mathrm{C}$ (Fig 3). $\omega 3 / \omega 6$ also exhibited an abrupt decline with SST, with the lowest values being reached at $22^{\circ} \mathrm{C}$, and had its intercept crossing at $19^{\circ} \mathrm{C}$. In addition, model predicted $\delta^{13} \mathrm{C}_{\text {corr }}$ (sexes combined or separate) and $\delta^{15} \mathrm{~N}_{\text {corr }}$ values in the muscle showed a rapid decline with SST with intercepts lying between $22^{\circ} \mathrm{C}$ and $24^{\circ} \mathrm{C}$ (Fig 4).

Chla influenced muscle NCIs and FATMs with intercept identifying thresholds in the range 0.3-0.5 $\mathrm{mg} \mathrm{m}^{-3}$ (Fig 3). TFA exhibited a linear increase with Chla while $\omega 3 / \omega 6$ and corrected SIs (Fig 3 and 4) showed a steep increase with Chla until around $1.4 \mathrm{mg} \mathrm{m}^{-3}$ after which they appeared to decrease slightly. On the other hand, DHA/EPA and $\omega 6$ protists FATM appeared to show the opposite trend and declined with Chla (Fig 3). 

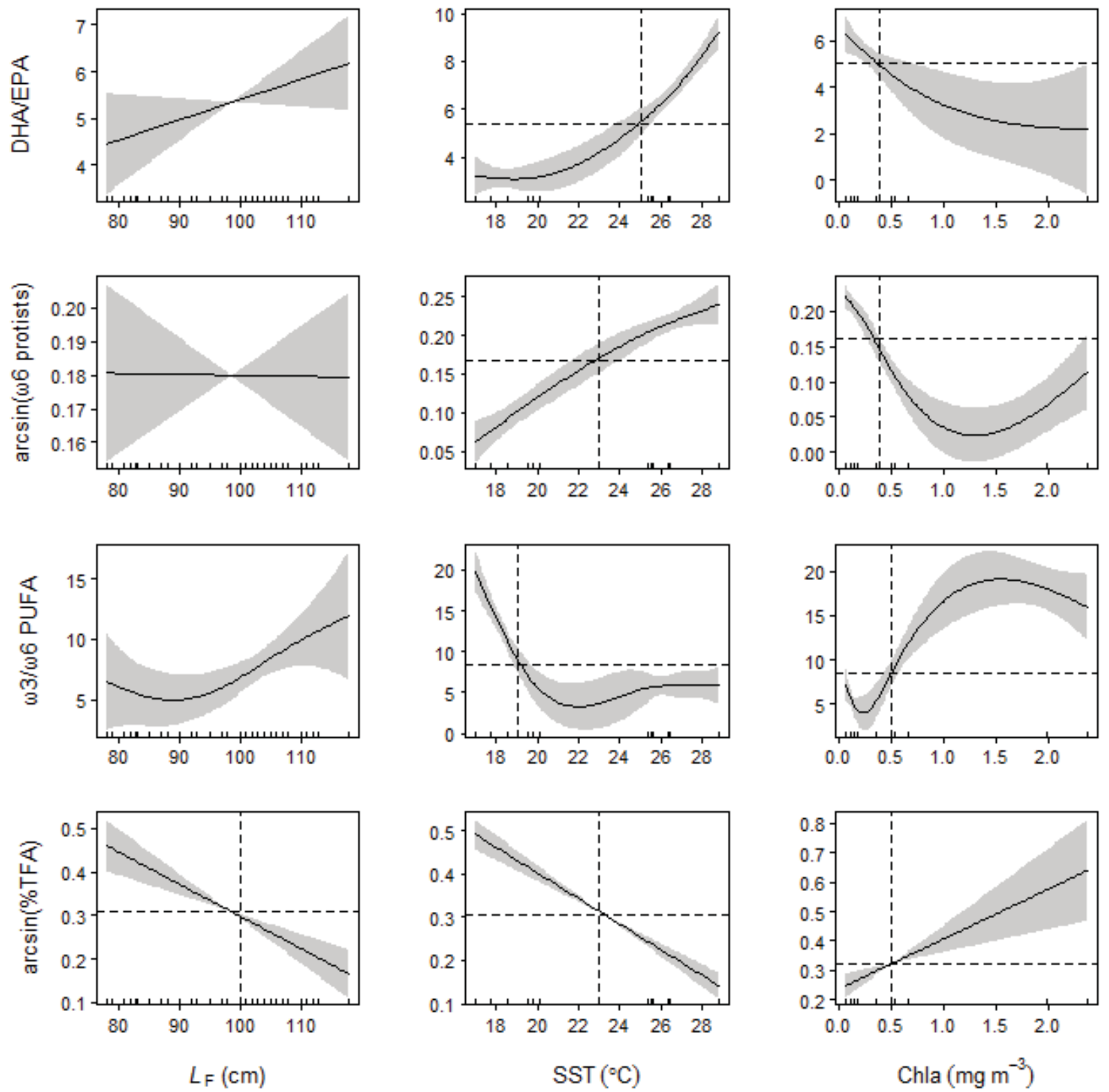

Fig 3. Smoother plots for GAMM-predicted docosahexaenoic acid/eicosapentaenoic acid (DHA/EPA), arcsinetransformed omega-6 (ळ6) protists fatty acid trophic marker and the nutritional condition indices (omega3/omega-6 polyunsaturated fatty acid, $\omega 3 / \omega 6$ PUFA, and arcsine-transformed \% total fatty acid content, TFA) in the muscle of male albacore tuna from the western Indian Ocean. The horizontal dashed lines in each plot represent the intercept while the vertical dashed lines demarcate the range of the variable above the zero line used as thresholds for significant GAMMs. The solid areas illustrate the confidence limits of the model which are twice the standard error. 

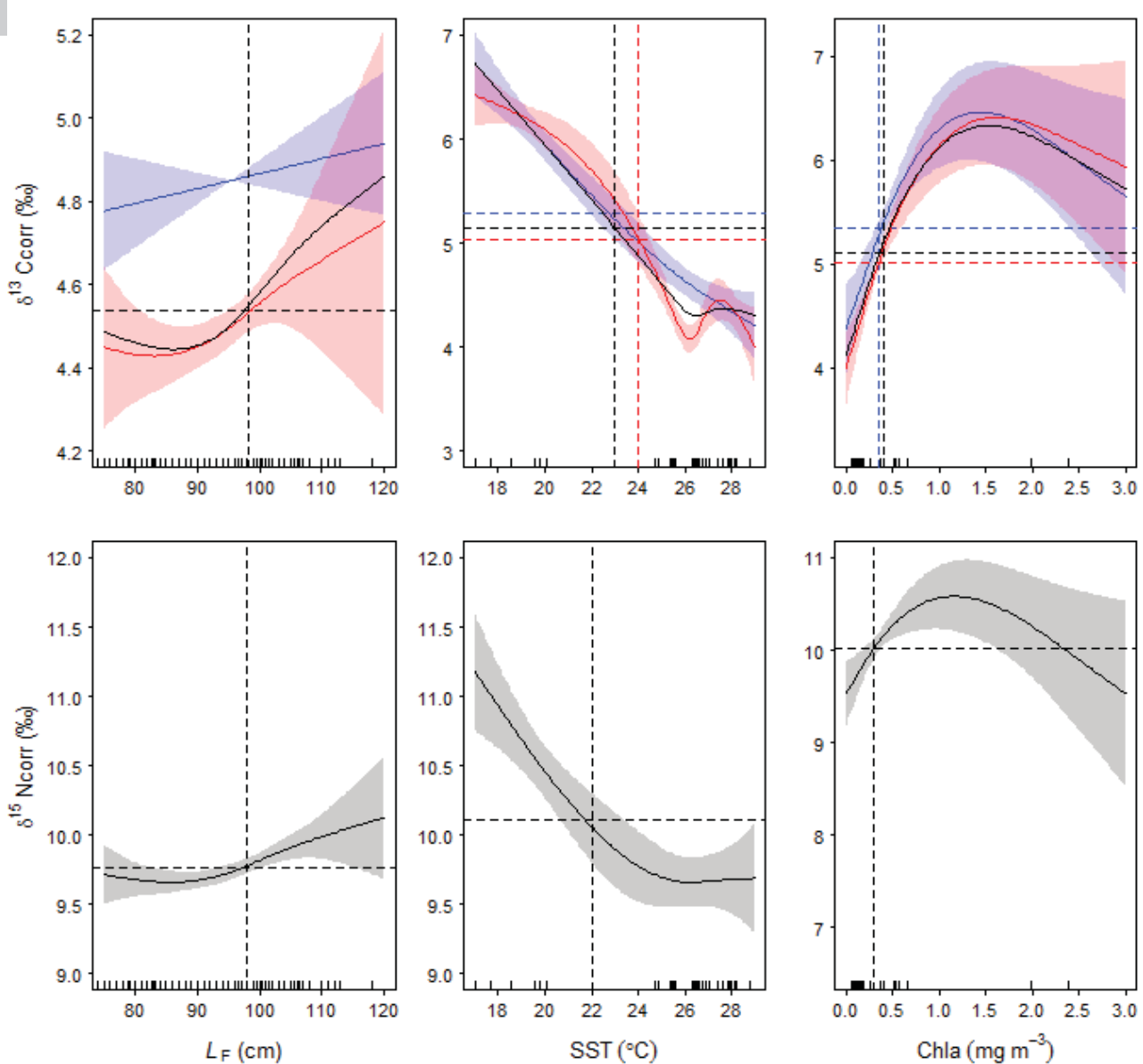

Fig 4. Smoother plots for GAMM-predicted corrected stable isotopes of carbon ( $\delta^{13} \mathrm{C}_{\text {corr, }} \%$ o) in male (blue) and female (red) albacore tuna in the western Indian Ocean, and nitrogen $\left(\delta^{15} \mathrm{~N}_{\text {corr }}\right.$, \%) for both sexes combined (black). The horizontal dashed lines in each plot represent the intercept while the vertical dashed lines demarcate the range of the variable above the zero line used as thresholds for significant models. The solid areas illustrate the confidence limits of the model which are twice the standard error. (Colour to be used in print)

For the FATMs and NCIs, the model with t(latitude, longitude) on its own was not significant $(P>0.05)$, except for DHA/EPA and TFA where it explained 81.0\% and 73.9\%, respectively, of the variability (SI1 Table). For all the corrected SI values, the model with t(latitude, longitude) was significant explained by deviance 68.1-91.0\%. The latitudinal variations of the trophic tracers and NCI, for which the model with t(latitude, longitude) was significant, are shown in Fig 5. However, male and female albacore were combined to illustrate latitudinal variation of $\delta^{13} \mathrm{C}_{\text {corr }}$ since both sexes were similarly affected by environmental parameters. TFA and corrected SIs reached higher values in the muscle of albacore caught south of $30^{\circ} \mathrm{S}$ (i.e., region $\mathrm{D}$ ) compared to those from latitudes north of $30^{\circ} \mathrm{S}$ (i.e., regions $\mathrm{A}, \mathrm{B}, \mathrm{C} 1$ and C2). DHA/EPA showed the opposite trend to that of the other trophic tracers and TFA as it clearly had highest values north of $30^{\circ} \mathrm{S}$, especially in Region A. 

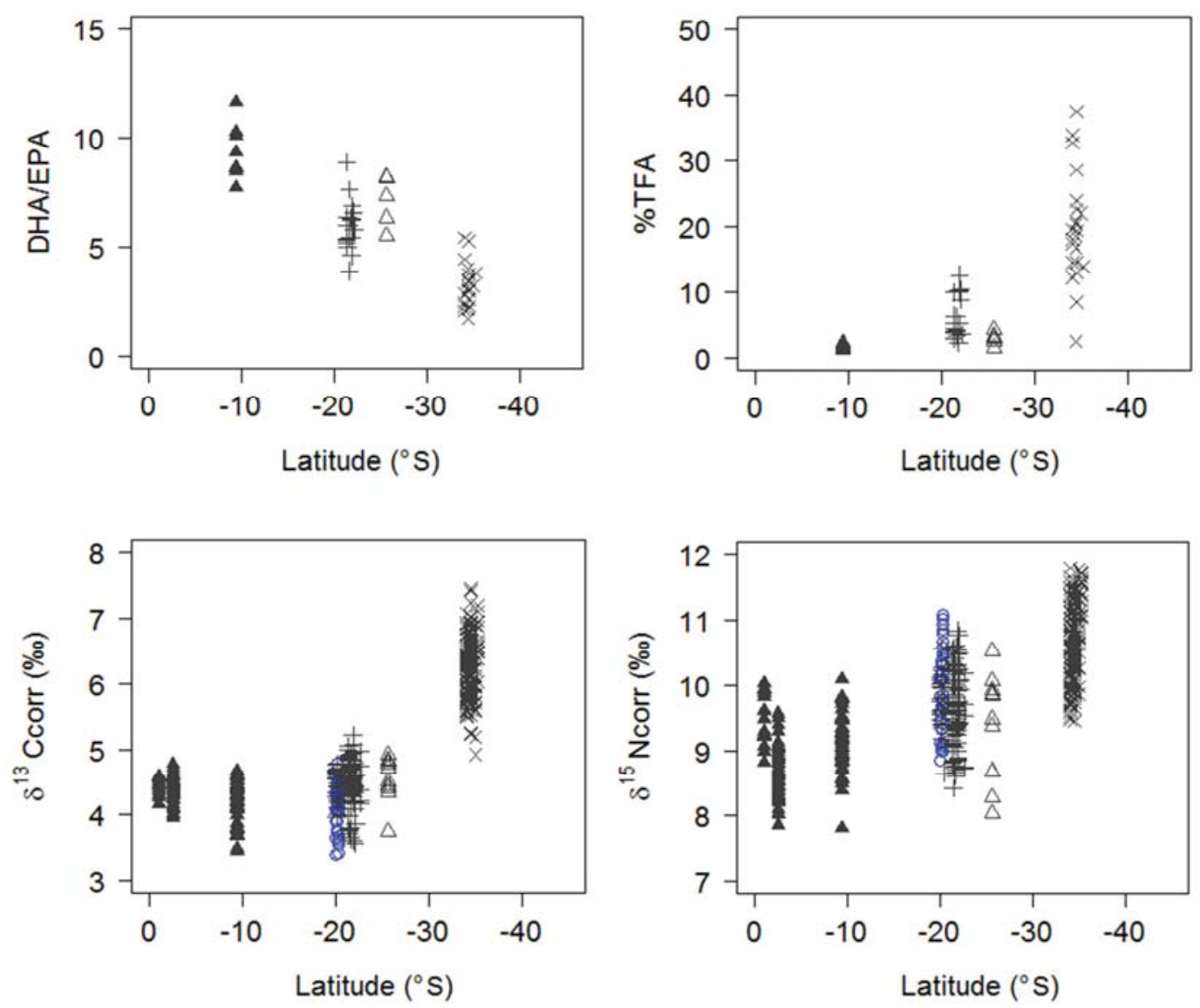

Fig 5. Plots of docosahexaenoic acid/eicosapentaenoic acid (DHA/EPA), \% total fatty acid content (TFA) and values of corrected stable isotopes of carbon $\left(\delta^{13} \mathrm{C}_{\text {corr }}, \%\right)$ and nitrogen $\left(\delta^{15} \mathrm{~N}_{\text {corr }}, \%\right)$ in the muscle tissue of albacore tuna, caught in different regions of the western Indian Ocean, against latitude. Regions A: Seychelles, B: Mozambique Channel, C1: Mauritius, C2: Reunion Island and D: South Africa. (Color to be used in print)

\section{Discussion}

To our knowledge, our study is the first to use NFAs and baseline-corrected SIs to examine the trophodynamics and nutritional condition of albacore caught in the western Indian Ocean. We found apparent differences in both FATMs and NCIs of albacore between the tropical (i.e., the waters of Seychelles, Mozambique Channel, Mauritius and Reunion Island) and temperate areas (South Africa) of the studied region. Our results reveal that FATMs can help differentiate regional and habitat usages by albacore as explained by changes in environmental conditions that impact the base of the food web and albacore life-history stages linked to migrations. At the same time, we identified the importance of diatoms in maintaining a high productivity in temperate waters and thus more efficient food webs (i.e., high $\delta^{13} \mathrm{C}_{\text {corr, }} \delta^{15} \mathrm{~N}_{\text {corr }}$ and low DHA/EPA) in which albacore can maintain a higher nutritional condition. The western Indian Ocean may be viewed as a hotspot in which SST is increasing at a faster rate than in other regions (Dong et al., 2014; Du and Xie, 2008). In the northern hemisphere, a reduction in the size of phytoplankton has been linked to the expansion of oligotrophic waters (Polovina and Woodworth, 2012) with increasing dinoflagellates in association with regime shifts (Möllmann and Diekmann, 2012). Even if both 
diatoms and dinoflagellates have comparable nutritional requirements, dinoflagellates may have an advantage over diatoms as they do not require silica and have ecological strategies that can promote their exploitation of coastal waters worldwide (Smayda, 2002). With the continued rise in SST of western Indian Ocean, dinoflagellates from the tropical areas could extend southward and potentially change the structure of the food web. Such changes may in turn favor tropical predators at the expense of temperate ones, including albacore (Polovina et al., 2009). In the northern hemisphere, albacore have been observed to modify their migration pattern as a response to a regime shift triggered by climate change (Dufour et al., 2010). In this respect, the detection of spatial patterns in ecological traits of albacore is essential for proper management of this important tuna species particularly in areas where oceanographic conditions are changing and seawater temperatures are warming faster than previously thought (Cheng et al., 2019).

\subsection{Variations in fatty acid trophic markers and nutritional condition}

The proportions of FATMs and NCIs in albacore muscle were shown to be highly responsive to environmental parameters. Both NCIs declined with increased SST and decreasing productivity while DHA/EPA showed the opposite trend (i.e. increased dinoflagellates). Through its direct impact on enzyme activity, temperature can affect biochemical and metabolic processes, including fatty acid synthesis, in both primary producers (Renaud et al., 1995; Thompson et al., 1992) and fish (Tocher and Sargent, 1990). A rise of 3-5\% in TFA and EPA was observed in cultured diatoms when temperature was decreased from $25^{\circ} \mathrm{C}$ to $10^{\circ} \mathrm{C}$ for a period of 12 hours (Jiang and Gao, 2004). Such changes in the fatty acid composition of primary producers can be observed along the food chain in consumers, as in the case of the western Indian Ocean albacore. Previous studies on the Pacific albacore have also demonstrated a strong effect of SST on the geographic variations of muscle fatty acids (Parrish et al., 2015; Pethybridge et al., 2015a). The results from the latter studies are in agreement with the distribution of

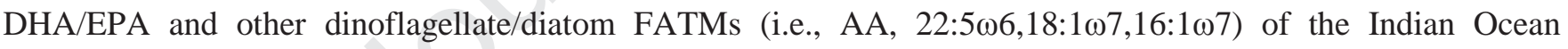
albacore which had a food web based mostly on dinoflagellates in tropical areas but on diatoms in temperate waters. Indeed, Sonnekus et al. (2017) described a latitudinal change in the phytoplankton community with low diversity and a change in dominance from dinoflagellates in the tropical regions to diatoms (mostly of genus Pseudo-nitzschia) towards the lower latitudes in the western Indian Ocean. High nutrient levels, which have been associated with temperatures less than $17.5^{\circ} \mathrm{C}$ (Sonnekus et al., 2017), favor the growth of large phytoplankton, including diatoms (Veldhuis et al., 1997). The southwest Indian Ocean has been described as a highly dynamic hydrographic region (Beal et al., 2011; Read et al., 2000). Along the eastern coast to the south of South Africa, the fast Agulhas Current flows west toward the Cape Point down to the Agulhas Bank off southern Africa, where its thermocline slope induces upwelling (Tomczak and Godfrey, 2003) thereby increasing productivity. On the other hand, the tropical waters of Seychelles, Reunion Island and Mauritius (regions A, C1 and C2) are more oligotrophic (Oliver and Irwin, 2008). Yet, during SWM, strong upwelling associated with the Ekman circulation create large areas with high nutrient concentration which then encourage phytoplankton blooms along the Somalian coast and other areas of the northern Indian Ocean (Veldhuis et al., 1997). As only fish from one season (SIM) were sampled and analyzed for their NFA profile in Seychelles and the Mozambique Channel (regions A and B), the effects of strong upwelling and eddies, and where diatoms were found to dominate in patchy blooms 
(Barlow et al., 2014; Veldhuis et al., 1997) in these regions, might have been missed in albacore muscle tissue. Instead, due to tissue turnover, FATMs reflected mostly the occurrence of picoplankton that usually dominate the phytoplankton community in the Mozambique Channel (Barlow et al., 2014) and during inter-monsoon periods in the northwest Indian Ocean (Owens et al., 1993).

The decline in TFA and $\omega 3 / \omega 6$ with increased SST, and the latitudinal variation of $\omega 3 / \omega 6$, observed in our study were comparable to that found in albacore from the south west Pacific Ocean where the same NCIs were used as indicators of health and productivity (Pethybridge et al., 2015a). Since lipids contain more energy in comparison to protein and carbohydrates, a decline in the TFA content will negatively affect the energy available to albacore, which may consequently affect their maintenance, growth and reproduction. This may specifically affect albacore which use a capital-income breeder strategy, and relying mostly on stored energy rather than concurrent feeding for reproduction (Dhurmeea et al., 2018). Marine organisms have been found to require long-chain PUFAs for early life development (Bell et al., 1995; Mourente et al., 1991). The right proportion of $\omega 3 / \omega 6$ is also a prerequisite as $\omega 6$ and $\omega 3$ were shown to exert an inhibitory bioconversion effect on each other (Watanabe, 1982). A high ratio of $\omega 3 / \omega 6$ seemed to be required for successful reproduction in tunas as observed in the ovaries of female albacore in the spawning capable phase in the western Indian Ocean (Dhurmeea et al., 2018). Since spawning of albacore occurs in tropical waters where this ratio was found to be lower in the muscle tissue during the present study, the importance of albacore in accumulating a high $\omega 3 / \omega 6$ in its tissues during their stay in temperate waters for feeding, as previously suggested by Dhurmeea et al. (2018), may be a prerequisite for future successful reproduction. Therefore, declines in long-chain PUFAs availability, more specifically, that of $\omega 3$ relative to $\omega 6$, as a result of climate change, is likely to negatively affect the performance and population dynamics of albacore populations.

Fish size was found to significantly influence the projected values of TFA. The size effect appears to have a threshold at approximately $98 \mathrm{~cm} L_{\mathrm{F}}$ which is around the size $(94 \mathrm{~cm})$ of the largest immature western Indian Ocean albacore (Dhurmeea et al., 2016b). Based on the length at 50\% maturity $\left(L_{50}\right)$ calculated from the latter and many other studies, most of the albacore caught from the waters of Seychelles, Mozambique Channel, Mauritius and Reunion Island were large and mature while those from South Africa (region D) consisted of a mixture of numerous small immature and adult individuals, particularly during SIM. The observed differences of FATMs and NCIs with fish size appear to be tightly linked to the occurrence of large mature albacore in tropical waters while younger immature ones are more restricted to the cooler temperate areas south of $30^{\circ} \mathrm{S}$ (Chen et al., 2005; Dhurmeea et al., 2016b; Suda, 1974) where they form part of a food web based mostly on diatoms. Large fish from South Africa seem to show intermediate proportions of FATMs between tropical-caught albacore and resident immature individuals from South Africa, in their muscle tissue. This may reflect either a recent migration of large fish to this region, after the spawning season, or continuous movement between the temperate South African and the tropical waters.

As concluded by Pethybridge et al. (2015a), variation of FATMs in albacore may also be related to a combination of interrelated factors such as ontogenetic change in diet (Young et al., 2001), faster metabolic rates of younger 
individuals and the trade-off between growth and reproduction in fishes (Claramunt et al., 2007). Further information through the combined approach of stomach content and prey fatty acid profile analyses of mature and immature albacore in the Indian Ocean can provide further insights into the trophic dynamics of their population. Such biochemical-stomach content analysis (SCA) combined approach can highly improve our understanding on trophic relationships (Annasawmy et al., 2018; Ménard et al., 2014).

\subsection{Variations in corrected stable isotope values}

We found that both $\delta^{13} \mathrm{C}_{\text {corr }}$ and $\delta^{15} \mathrm{~N}_{\text {corr }}$ values declined with increasing SST and decreasing productivity (Fig 4) which is similar to the observed variations for the NCIs but opposite to DHA/EPA and $\omega 6$ protists FATM (Fig 3). Moreover, just as the FATMs and NCIs, we observed a strong latitudinal effect in corrected SI, as shown by the increasing southward pattern in the $\delta^{13} \mathrm{C}_{\text {corr }}$ values and $\delta^{15} \mathrm{~N}_{\text {corr }}$ values (Fig 5). As inferred from the GAMMs, $\delta^{13} \mathrm{C}_{\text {corr }}$ values are highly influenced by latitude while $\delta^{15} \mathrm{~N}_{\text {corr }}$ values are mostly driven by SST. Our analysis also shows the effect of month on $\delta^{13} \mathrm{C}_{\text {corr }}$ values which were lower during NEM and could be linked to the seasonal migration of tunas from the temperate to tropical areas during this season for spawning (Dhurmeea et al., 2016b). Several trophic studies have shown that $\delta^{13} \mathrm{C}$ varied strongly with latitude contrary to $\delta^{15} \mathrm{~N}$ which responded mostly to trophic enrichment (Cherel and Hobson, 2007; Quillfeldt et al., 2005). SI values of predators reflect those of their prey which is in turn dependent on the isotopic signature at the base of the food web (Fry, 2006; Post, 2002). Yet, similarly to fatty acids, muscle SI values do not reflect that of their recent prey due to the long tissue turnover rate. In migratory species, the isotopic signature will be affected by both the regional SI composition of the sampling area and conditions of the area that it inhabited earlier (Hansson et al., 1997). Those individuals that are more resident in one area would have SI patterns reflecting that of the base of the food web (Fry, 2006; Ménard et al., 2007). The combination of these effects creates variability in SIs and may blur their interpretation as trophic tracers. This can be observed in the $\delta^{13} \mathrm{C}$ values in albacore muscle without adjusting for baseline variability (Fig 6). Adjusting for baseline variability may help to overcome this issue but requires careful interpretation as it may not reflect changes observed in other trophic studies using SIs. For instance, $\delta^{13} \mathrm{C}$ was found to increase in the tissues of consumers with increasing latitude, such as in the Indian Ocean yellowfin tuna and swordfish (Ménard et al., 2007; Zudaire et al., 2015), and the Pacific albacore tuna (Pethybridge et al., 2015b). Indeed, $\delta^{13} \mathrm{C}$ of both phytoplankton and albacore muscle tissue (without baseline correction) in our study showed the same trend to the above studies. This may possibly suggest that the variation of $\delta^{13} \mathrm{C}$ at the base of the food chain influences that of consumers and may not reveal the actual assimilation of carbon into the tissue of consumers throughout the various trophic levels along the food web, especially if the consumer is a highly migratory species, spending various part of its life cycle in areas characterized by different seawater isotopic composition. Tissue $\delta^{13} \mathrm{C}$ of tunas is subject to such a large variability that it can even be used for investigating variations in atmospheric $\mathrm{CO}_{2}$ and anthropogenic climate changes (Lorrain et al., 2019). 

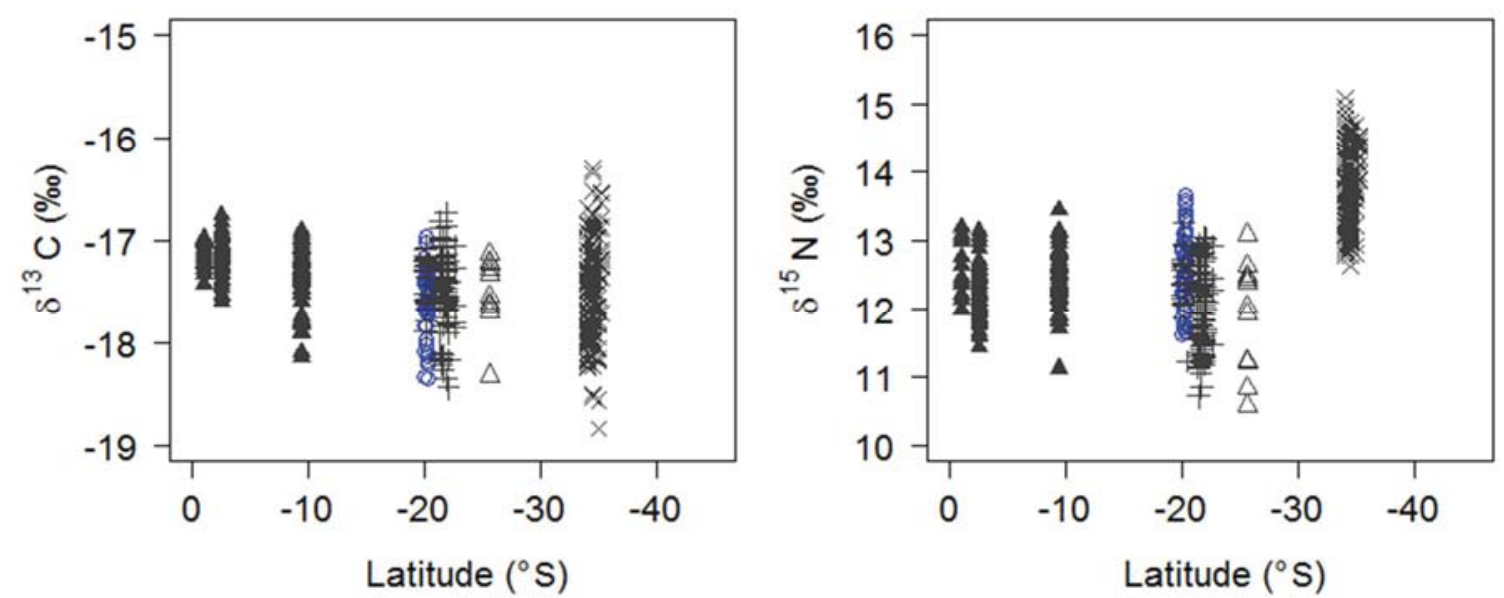

Fig 6. Plots of lipid-corrected carbon $\left(\delta^{13} \mathrm{C}\right.$, \%) and nitrogen $\left(\delta^{15} \mathrm{~N}, \%\right)$ stable isotope values in the muscle tissue of albacore tuna, caught in different regions of the western Indian Ocean, against latitude. Regions A: Seychelles, B: Mozambique Channel, C1: Mauritius, C2: Reunion Island and D: South Africa. (Color to be used in print)

\subsection{Variations in trophic position and dietary linkages}

We observed an increase in TP, as derived from $\delta^{15} \mathrm{~N}_{\text {corr }}$ values, with decreasing latitude from the tropical regions (4.9) towards the temperate waters of region D (5.4), such that three distinct groups of albacore, characterized by different TP could be identified: albacore from (i) Seychelles and Mozambique Channel (regions A and B) (ii) Mauritius and Reunion Island (regions C1 and C2), and (iii) South Africa (region D). The low TP of albacore prevailing in Seychelles and Mozambique Channel could be attributed to intense foraging of prey at lower TP, for instance, the planktophage cigarfish, Cubiceps pauciradiatus, which is known to occur in abundance in the regions of Seychelles and Somalia, especially during NEM and SWM (Potier et al., 2008; Romanov et al., 2009; Schott et al., 2002). Cigarfish has been described as an important prey item for yellowfin tuna in the region of Seychelles (Zudaire et al., 2015). In contrast, in the region of the South Subtropical Gyre, which includes both the waters of regions Mauritius and Reunion Island, the importance of cephalopods as a prey for albacore was demonstrated with a high occurrence of squids and octopus (Dhurmeea et al., in press; Romanov et al., in press). Squids have been described to range from medium to top trophic levels in marine food webs (Amaratunga, 1983; Coll et al., 2013) in comparison to myctophid fishes which feed at lower TP (Cherel et al., 2010; Koz, 1995; Young et al., 2006). Thus, feeding on prey found at higher TPs could be one of the reasons accounting for the relatively high $\delta^{15} \mathrm{~N}_{\text {corr }}$ values and TP of albacore in regions of Mauritius and Reunion Island.

However, studies based on SCA need to be interpreted carefully as they only reflect the most recent meal and may not represent the actual diet (Das et al., 2000). SCA also requires a large number of samples to capture spatial and temporal variations of the diet (Ménard et al., 2007). This is particularly crucial in the case of highly migratory species like tunas. Moreover, the length of a food chain and the relative TP of a consumer that forages in different 
regions can be affected by the complexity of the food webs that prevail in these different regions (Jennings et al., 2008; Vander Zanden and Fetzer, 2007). This may be because factors that can affect $\delta^{15} \mathrm{~N}$ values of a predator are not only the number of trophic levels before that of the predator in a food web but may also involve isotopic baseline variations (Lorrain et al., 2015; Vander Zanden and Rasmussen, 2001). High primary productivity may increase a consumer's TP by supporting large prey densities (Olsson et al., 2008). For instance, it was recently shown that $\delta^{15} \mathrm{~N}$ values and TP of micronekton in the western Indian Ocean varied between the oligotrophic regions of the South Subtropical Gyre and the more mesotrophic east African coast with higher values found in both mesopelagic fishes, squid and crustaceans in the latter area (Annasawmy et al., 2018). This may in turn account for the high TP observed in albacore caught in South African waters. High productivity in this region is in accordance to the increased concentrations of oxygen, nitrate and nitrite, and phytoplankton biomass in the waters from the lower latitudes of the western Indian Ocean (Sonnekus et al., 2017), potentially being part of the subAntarctic surface water (Pollard and Read, 2017), and the associated FATMs indicative of a diatom-based food web observed in this region. In contrast, low TP in the other regions of the western Indian Ocean, within the South Subtropical Gyre, may be linked to their more oligotrophic nature (Chouvelon et al., 2017; Longhurst, 2006) usually characterized by low Chla concentrations, up to $0.1 \mathrm{mg} \mathrm{m}^{-3}$ (Vecsei, 2003) and where nitrate is the major limiting factor (Moore et al., 2013) resulting in lower particle organic matter $\delta^{15} \mathrm{~N}$ values. The oligotrophy is especially pronounced during inter-monsoon periods (SIM and AIM) when the Indian Ocean is stratified with very low Chla concentrations $\left(<0.2 \mu \mathrm{l}^{-1}\right)$ in surface waters thereby decreasing the productivity (Veldhuis et al., 1997). Our observation of the rising trend of the corrected SI values in the muscle tissue of albacore with Chla is also therefore consistent with the fact that high Chla concentrations $\left(>0.2 \mathrm{mg} \mathrm{m}^{-3}\right)$, characteristic of mesotrophic waters (Vecsei, 2003), are associated with high levels of inorganic nitrogen (Polovina et al., 2001) leading to an enrichment of zooplankton with ${ }^{15} \mathrm{~N}$ (Mullin et al., 1984). In comparison to tropical tunas that prefer warm and anoxic waters, temperate tunas have a preference for habitats rich in chlorophyll (Arrizabalaga et al., 2015; Duffy et al., 2017). In fact, juvenile albacore were observed to be associated with chlorophyll fronts (with concentrations of around $0.2 \mathrm{mg} \mathrm{m}^{-3}$ ) exploiting them during their migration route across the Pacific Ocean as they feed on prey which themselves forage along these fronts (Polovina et al., 2001).

The estimated TP for albacore in our study (4.25-5.92) was higher compared to that of adult bluefin tuna, Thunnus thynnus (4.1), yellowfin, juvenile bluefin and albacore tunas (3.2-3.6) from the North Atlantic (Estrada et al., 2005). Apart from the probable complexity in food web in different regions, these differences may also originate from assumptions when estimating TP, such as the different baseline values and TEF used by different authors. The assumption of a TEF of around 3\% per TP is not consistent among consumers and may lead to errors (Caut et al., 2009; Hussey et al., 2014; Vanderklift and Ponsard, 2003). Through this study, one method to adjust for baseline variability effects from tissue $\delta^{15} \mathrm{~N}$ signature was utilized. Despite these possible variations in TP, the fact that, in the western Indian Ocean region, crustacean, fish and squid micronekton, which form the link between zooplanton and large predatory fishes (Potier et al., 2007), have been reported to be tertiary consumers with TP 2.6-4.2 (Annasawmy et al., 2018; Ménard et al., 2014), is in accordance with the observed TP of albacore in the present study. In addition, using baseline correction, TP similar to our results have been identified globally for albacore (4.5), yellowfin (4.7) and big eye (5.1) tunas (Pethybridge et al., 2018). 


\subsection{Variations in lipid and baseline corrected stable isotope of carbon}

$\delta^{13} \mathrm{C}$ values of phytoplankton are expected to decrease from low to high latitudes (Lourey et al., 2003) due to variations in the $\delta^{13} \mathrm{C}$ gradient in particulate organic matter of surface waters which are usually higher in the northern warm subtropical waters but lower in colder Antarctic waters (Francois et al., 1993; Trull and Armand, 2001). Indeed, estimated $\delta^{13} \mathrm{C}$ values of phytoplankton were higher in the regions of Seychelles, Mauritius and Reunion Island, but the adjustment for baseline variability (through the use of $\delta^{13} \mathrm{C}_{\text {corr }}$ ) led to different results. Instead, we found that $\delta^{13} \mathrm{C}_{\text {corr }}$ values were higher in the temperate South African waters. High $\delta^{13} \mathrm{C}$ values in consumers may also occur in temperate waters if the region that they prevail falls within the Subtropical, Subantarctic or Polar front, where abrupt increases in $\delta^{13} \mathrm{C}$ gradient in particle organic matter are known to occur (Cherel and Hobson, 2007; Ménard et al., 2007). Furthermore, in the Subtropical Convergence, peak particulate organic carbon (POC)- $\delta{ }^{13} \mathrm{C}$ was found to coincide with, not only peak particulate organic matter, but Chla as well (Francois et al., 1993). This could account for the observed relationships of $\delta^{13} \mathrm{C}_{\text {corr }}$ with Chla and SST in the present study where $\delta^{13} \mathrm{C}_{\text {corr }}$ was found to increase with the former but decrease with the latter. The high $\delta^{13} \mathrm{C}_{\text {corr }}$ values in albacore muscle tissue in South Africa region may thus reflect the assimilation of $\delta^{13} \mathrm{C}$ when albacore fed along these fronts in the more temperate regions. Besides, $\delta^{13} \mathrm{C}$ of a predator can be affected by the planktonic communities prevailing in a particular area. We found that diatoms had a larger contribution than dinoflagellates in the temperate waters of South Africa. Despite the fact that diatoms exhibit slower growth rates at low temperatures (Curl Jr and McLeod, 1961; Sakshaug, 1977), larger carbon fractionations have been observed (Fry, 1996). Phytoplankton utilizing bicarbonate as their carbon source increase the level of POC- $\delta^{13} \mathrm{C}$ while those taking up mainly dissolved carbon dioxide, decrease its level (Francois et al., 1993). In this way, the elevated $\delta^{13} \mathrm{C}_{\text {corr }}$ prevailing in the tissues of albacore from South African waters may be linked to the fact that diatoms use the heavy isotope of bicarbonate ion as a source of carbon and are thus usually heavier in their $\delta^{13} \mathrm{C}$ compared to flagellates (Fry and Wainright, 1991). Furthermore, POC from temperate regions characterized by high productivity have a tendency to be isotopically heavier and may be associated to diatoms' ability to rapidly assimilate nitrogen with increased uptake of bicarbonate (Wilkerson and Dugdale, 1987; Zimmerman et al., 1987). As such, diatoms go through a larger fractionation of carbon during photosynthesis and contribute to higher $\delta^{13} \mathrm{C}$ values (Lorrain et al., 2019) which upon grazing provide the bulk of the carbon in marine food webs in the temperate areas. Even small changes in phytoplankton community composition or physiology are suspected to influence tuna muscle $\delta^{13} \mathrm{C}$ values (Lorrain et al., 2019). This implies that a reduction in the abundance of diatoms as a result of climate change may negatively impact on $\delta^{13} \mathrm{C}$ values of consumers in marine food webs (Fry and Wainright, 1991). Yet, other factors such as nutritional status, physiological change (Focken and Becker, 1998; Lorrain et al., 2002) and growth rate (Olive et al., 2003) may affect $\delta^{13} \mathrm{C}$ in predators.

\subsection{Ontogenetic changes in stable isotopes}


For both $\delta^{13} \mathrm{C}_{\text {corr }}$ and $\delta^{15} \mathrm{~N}_{\text {corr }}$, length was a significant explanatory variable, particularly when both sexes were combined. Differences between small and large fish were observed whereby large individuals (>100 cm $L_{\mathrm{F}}$ ) exhibited higher SI values in their muscle tissue. Size difference in $\delta^{15} \mathrm{~N}_{\text {corr }}$ was noted in South African waters only where a high proportion of immature albacore individuals occurred (Dhurmeea et al., 2016b). Such variation may be linked to smaller albacore consuming mostly prey species at lower trophic levels while the larger fish could prey on larger and higher trophic level species, as similarly described for swordfish in the region (Ménard et al., 2007). For instance, large swordfish are known to have a preference for cephalopods while small swordfish preyed on lower trophic level species such as mesopelagic fishes (Potier et al., 2007; Young et al., 2006). Both SCA and nitrogen SI values in fish have demonstrated that both prey size and trophic level increase with increasing size of predators (Jennings et al., 2002; Scharf et al., 2000). Similar enrichment of $\delta^{15} \mathrm{~N}$ with size was observed in the Pacific albacore by Pethybridge et al. (2015b) as well as other species although large predators could still feed on small prey (Ménard et al., 2007; Young et al., 2010). The increase in $\delta^{15} \mathrm{~N}_{\text {corr }}$ with size may be linked to the increased diving ability of adult fish to access a wider range of prey (Sardenne et al., 2016). Juvenile albacore, less than $80-90 \mathrm{~cm} L_{\mathrm{F}}$, are thought to be less capable to perform vertical migration as their swim bladder is not yet functional (Gibbs and Collette, 1967). As adult albacore can dive below $400 \mathrm{~m}$ depth (Bertrand et al., 2002), their diet composition may therefore depend on their vertical behavior as seen in the Pacific albacore (Williams et al., 2015) which exhibited diel vertical behavior in tropical regions and had a higher diversity of prey in the stomach, consisting mainly of deep-water dwelling species. On the other hand, in temperate areas, albacore showed almost no vertical movement with individuals restricted to waters above the mixed layer depth (Williams et al., 2015). Compared to smaller individuals, larger predators may additionally be able to catch larger prey, characterized by higher $\delta^{15} \mathrm{~N}$ (Parry, 2008), from their larger mouth-gape and higher chasing ability (Carey and Robinson, 1981). The increase in the SI of carbon has also been reported in the Pacific bluefin, Thunnus orientalis (Madigan et al., 2012), and albacore tunas (Pethybridge et al., 2015b) which was in turn associated to the variations in feeding with size and/or age as described for tropical tunas (Sardenne et al., 2016) where smaller individuals fed at shallower depths compared to adults. Another factor that may affect $\delta^{13} \mathrm{C}_{\text {corr }}$ is the faster metabolic rate of young albacore resulting in higher isotopic turnover rates in comparison to adults (MacAvoy et al., 2006).

\section{Conclusion}

Our biochemical tracer approach using predictive models revealed the effects of fish length, geographic location and environmental parameters on NFA and SI trophic tracers of the albacore from the western Indian Ocean. Their variability was also attributed to the life-history stage distribution of albacore with smaller and younger individuals inhabiting the South Africa waters having different isotopic and NFA signatures compared to the larger albacore. Nutritional condition was lower in albacore from the tropical than temperate waters, suggesting that increased SST as a result of climate change could negatively affect population dynamics and nutritional quality of albacore. Both SI and NFA trophic tracers agree and confirm that changes in SST and productivity will impact nutrient flow and energy transfer in the marine food web. Furthermore, we found that $\delta^{15} \mathrm{~N}_{\text {corr }}$ (affected by prey consumption) was effective at defining trophic relationships tissue while $\delta^{13} \mathrm{C}_{\text {corr }}$ (determined by 
geographical position) could be used to track movement patterns. Further analyses, covering both a maximum number of seasons and other areas, including the central and eastern part of the Indian Ocean, as well as the southeast Atlantic, would be required to fully assess their regional variation. In particular, analyzing additional small albacore individuals $\left(<70 \mathrm{~cm} L_{\mathrm{F}}\right.$ ), found within the southern temperate waters, would be needed to better understand the sensitivity of the albacore population to global changes. Future studies should also focus on the examination of the NFA profiles of prey of albacore in various regions of the western Indian Ocean.

\section{Acknowledgments}

The present work was undertaken under the framework of the EU FEP 2007-2013 project GERMON (NÊ759/DMSOI/2013) and the EU DCF project (Reg. 199/2008 and 665/2008). We would like to thank various people for their help during sampling: J. Esparon, A. Tirant, A. Stephen, R. Rose, E. Mathiot, M. Elisabeth, P. Boniface, M. Lesperance, and S. Hollanda from SFA; M. Meyer, J. Cunningham, W. West and K. Baloyi from DAFF and C. Heinecken from Capfish; E. Romanov, E. Richard and L. Le Foulgoc from CAP RUNĐHYDRORUN, and finally H. Evano, A. Puech and D. Roos from Ifremer DOI La Réunion. We thank the fishermen, skippers and other members of the fishing industry including the workers from the tuna processing factories and canneries in Seychelles (Indian Ocean Tuna Ltd), Mauritius (Pelagic Process Ltd), South Africa (Indian Ocean Tuna Ltd) and Reunion Island (Reunipeche and Manohal team) for their cooperation and assistance during sampling activities.

Funding: This work is part of the $\mathrm{PhD}$ of the first author and was supported by the "Allocations de Recherche pour une Thèse au Sud" (ARTS) program of the French Institute of Research for Development (IRD).

\section{Declarations of interest: none}

\section{References}

Amaratunga, T., 1983. The role of cephalopods in the marine ecosystem, in: Caddy, J.F. (Ed.), Advances in Assessment of World Cephalopod Resources. FAO Fisheries Technical Paper, pp. 379-415.

Annasawmy, P., Ternon, J.-F., Marsac, F., Cherel, Y., Béhagle, N., Roudaut, G., Lebourges-Dhaussy, A., Demarcq, H., Moloney, C.L., Jaquemet, S., 2018. Micronekton diel migration, community composition and trophic position within two biogeochemical provinces of the South West Indian Ocean: Insight from acoustics and stable isotopes. Deep Sea Res. Part Oceanogr. Res. Pap. 138, 85-97.

Arrizabalaga, H., Dufour, F., Kell, L., Merino, G., Ibaibarriaga, L., Chust, G., Irigoien, X., Santiago, J., Murua, H., Fraile, I., Chifflet, M., Goikoetxea, N., Sagarminaga, Y., Aumont, O., Bopp, L., Herrera, M., Marc Fromentin, J., Bonhomeau, S., 2015. Global habitat preferences of commercially valuable tuna. Deep Sea Res. Part II Top. Stud. Oceanogr. 113, 102-112.

Auel, H., Harjes, M., Da Rocha, R., Stübing, D., Hagen, W., 2002. Lipid biomarkers indicate different ecological niches and trophic relationships of the Arctic hyperiid amphipods Themisto abyssorum and T. libellula. Polar Biol. 25, 374-383.

Barlow, R., Lamont, T., Morris, T., Sessions, H., van den Berg, M., 2014. Adaptation of phytoplankton communities to mesoscale eddies in the Mozambique Channel. Deep Sea Res. Part II Top. Stud. Oceanogr., The Mozambique Channel: Mesoscale Dynamics and Ecosystem Responses 100, 106-118.

Beal, L.M., De Ruijter, W.P., Biastoch, A., Zahn, R., Cronin, M., Hermes, J., Lutjeharms, J., Quartly, G., Tozuka, T., Baker-Yeboah, S., 2011. On the role of the Agulhas system in ocean circulation and climate. Nature 472, 429.

Bell, M.V., Batty, R.S., Dick, J.R., Fretwell, K., Navarro, J.C., Sargent, J.R., 1995. Dietary deficiency of docosahexaenoic acid impairs vision at low light intensities in juvenile herring (Clupea harengus L.). Lipids 30, 443-449. 
Bertrand, A., Bard, F.-X., Josse, E., 2002. Tuna food habits related to the micronekton distribution in French Polynesia. Mar. Biol. 140, 1023-1037.

Bodin, N., Budzinski, H., Le Ménach, K., Tapie, N., 2009. ASE extraction method for simultaneous carbon and nitrogen stable isotope analysis in soft tissues of aquatic organisms. Anal. Chim. Acta 643, 54-60.

Burnham, K.P., Anderson, D.R., 2004. Multimodel inference: understanding AIC and BIC in model selection. Sociol. Methods Res. 33, 261-304.

Carey, F.G., Robinson, B.H., 1981. Daily patterns in the activities of swordfish, Xiphias gladius, observed by acoustic telemetry. Fish. Bull. 79, 277-292.

Caut, S., Angulo, E., Courchamp, F., 2009. Variation in discrimination factors $\left(\Delta^{15} \mathrm{~N}\right.$ and $\left.\Delta^{13} \mathrm{C}\right)$ : the effect of diet isotopic values and applications for diet reconstruction. J. Appl. Ecol. 46, 443-453.

Chen, I.-C., Lee, P.-F., Tzeng, W.-N., 2005. Distribution of albacore (Thunnus alalunga) in the Indian Ocean and its relation to environmental factors. Fish. Oceanogr. 14, 71-80.

Cheng, L., Abraham, J., Hausfather, Z., Trenberth, K.E., 2019. How fast are the oceans warming? Science 363, 128-129.

Cherel, Y., Fontaine, C., Richard, P., Labatc, J.-P., 2010. Isotopic niches and trophic levels of myctophid fishes and their predators in the Southern Ocean. Limnol. Oceanogr. 55, 324-332.

Cherel, Y., Hobson, K.A., 2007. Geographical variation in carbon stable isotope signatures of marine predators: a tool to investigate their foraging areas in the Southern Ocean. Mar. Ecol. Prog. Ser. 329, 281-287.

Chouvelon, T., Brach-Papa, C., Auger, D., Bodin, N., Bruzac, S., Crochet, S., Degroote, M., Hollanda, S.J., Hubert, C., Knoery, J., 2017. Chemical contaminants (trace metals, persistent organic pollutants) in albacore tuna from western Indian and south-eastern Atlantic Oceans: Trophic influence and potential as tracers of populations. Sci. Total Environ. 596, 481-495.

Claramunt, G., Serra, R., Castro, L.R., Cubillos, L., 2007. Is the spawning frequency dependent on female size? Empirical evidence in Sardinops sagax and Engraulis ringens off northern Chile. Fish. Res. 85, $248-257$.

Coelho, R., Nikolic, N., Evano, H., Miguel, N., Bourjea, J., 2014. Reunion island pelagic longline fishery characterization and standardization of albacore catch rates. IOTC-2014-WPTmT05-12 Rev_1. Presented at the Fifth Working Party on Temperate Tunas, 28-31 July 2014, Busan, Rep. of Korea, Indian Ocean Tuna Commission, Seychelles.

Coll, M., Navarro, J., Olson, R.J., Christensen, V., 2013. Assessing the trophic position and ecological role of squids in marine ecosystems by means of food-web models. Deep Sea Res. Part II Top. Stud. Oceanogr. 95, 21-36.

Collette, B.B., Nauen, C.E., 1983. Scombrids of the words. FAO, Rome (Italy).

Curl Jr, H., McLeod, G.C., 1961. The physiological ecology of a diatoms, Skeletonema costatum (Grev.) Cleve. J. Mar. Res. 19, 70-88.

Dalsgaard, J., John, M.S., Kattner, G., Müller-Navarra, D., Hagen, W., 2003. Fatty acid trophic markers in the pelagic marine environment. Adv. Mar. Biol. 46, 225-340.

Das, K., Lepoint, G., Loizeau, V., Debacker, V., Dauby, P., Bouquegneau, J.-M., 2000. Tuna and dolphin associations in the North-East Atlantic: evidence of different ecological niches from stable isotope and heavy metal measurements. Mar. Pollut. Bull. 40, 102-109.

Dhurmeea, Z., Beeharry, S.P., Sooklall, T., 2012. Catch/effort and length-frequency data collected on albacore tuna landed in Mauritius (IOTC-2012-WPTmT04-12), in: Fourth Working Party on Temperate Tunas, 2023 August 2012, Shanghai, China. Indian Ocean Tuna Commission, Seychelles, p. 11.

Dhurmeea, Z., Chassot, E., Augustin, E., Assan, C., Nikolic, N., Bourjea, J., West, W., Appadoo, C., Bodin, N., 2016a. Morphometrics of albacore tuna (Thunnus alalunga) in the western Indian Ocean (IOTC-2016WPTmT06-28), in: Sixth Working Party on Temperate Tunas, 18-21 July 2016, Shanghai, China. Indian Ocean Tuna Commission, Seychelles, p. 19.

Dhurmeea, Z., Zudaire, I., Chassot, E., Cedras, M., Nikolic, N., Bourjea, J., West, W., Appadoo, C., Bodin, N., 2016b. Reproductive biology of albacore tuna (Thunnus alalunga) in the western Indian Ocean. Plos One 11, e0168605.

Dhurmeea, Z., Pethybridge, H., Appadoo, C., Bodin, N., 2018. Lipid and fatty acid dynamics in mature female albacore tuna (Thunnus alalunga) in the western Indian Ocean. PloS One 13, e0194558.

Dhurmeea, Z., Pethybridge, H., Romanov, E., Appadoo, C., Bodin, N., in press. Diet of albacore tuna, Thunnus alalunga, from the waters of Mauritius (western Indian Ocean) inferred by stomach content and fatty acid analyses.

Dong, L., Zhou, T., Wu, B., 2014. Indian Ocean warming during 1958-2004 simulated by a climate system model and its mechanism. Clim. Dyn. 42, 203-217.

Du, Y., Xie, S.-P., 2008. Role of atmospheric adjustments in the tropical Indian Ocean warming during the 20th century in climate models. Geophys. Res. Lett. 35. 
Duffy, L.M., Kuhnert, P.M., Pethybridge, H.R., Young, J.W., Olson, R.J., Logan, J.M., Goñi, N., Romanov, E., Allain, V., Staudinger, M.D., 2017. Global trophic ecology of yellowfin, bigeye, and albacore tunas: understanding predation on micronekton communities at ocean-basin scales. Deep Sea Res. Part II Top. Stud. Oceanogr. 140, 55-73.

Dufour, F., Arrizabalaga, H., Irigoien, X., Santiago, J., 2010. Climate impacts on albacore and bluefin tunas migrations phenology and spatial distribution. Prog. Oceanogr. 86, 283-290.

El-Sabaawi, R., Dower, J.F., Kainz, M., Mazumder, A., 2009. Characterizing dietary variability and trophic positions of coastal calanoid copepods: insight from stable isotopes and fatty acids. Mar. Biol. 156, 225237.

Estrada, J.A., Lutcavage, M., Thorrold, S.R., 2005. Diet and trophic position of Atlantic bluefin tuna (Thunnus thynnus) inferred from stable carbon and nitrogen isotope analysis. Mar. Biol. 147, 37-45.

Focken, U., Becker, K., 1998. Metabolic fractionation of stable carbon isotopes: implications of different proximate compositions for studies of the aquatic food webs using $\delta^{13} \mathrm{C}$ data. Oecologia 115, 337-343.

Francois, R., Altabet, M.A., Goericke, R., McCorkle, D.C., Brunet, C., Poisson, A., 1993. Changes in the $\delta^{13} \mathrm{C}$ of surface water particulate organic matter across the subtropical convergence in the SW Indian Ocean. Glob. Biogeochem. Cycles 7, 627-644.

Fry, B., 2006. Stable isotope ecology. Springer, New York.

Fry, B., 1996. 13C/12C fractionation by marine diatoms. Mar. Ecol. Prog. Ser. 134, 283-294.

Fry, B., Wainright, S.C., 1991. Diatom sources of ${ }^{13}$ C-rich carbon in marine food webs. Mar. Ecol. Prog. Ser. 149-157.

Furuita, H., Tanaka, H., Yamamoto, T., Suzuki, N., Takeuchi, T., 2002. Effects of high levels of n- 3 HUFA in broodstock diet on egg quality and egg fatty acid composition of Japanese flounder, Paralichthys olivaceus. Aquaculture 210, 323-333.

Gibbs, R.H., Collette, B.B., 1967. Comparative anatomy and systematics of the tunas, genus Thunnus. Fish. Bull. US 86, 835-838.

Hansson, S., Hobbie, J.E., Elmgren, R., Larsson, U., Fry, B., Johansson, S., 1997. The stable nitrogen isotope ratio as a marker of food-web interactions and fish migration. Ecology 78, 2249-2257.

Hobson, K.A., Piatt, J.F., Pitocchelli, J., 1994. Using stable isotopes to determine seabird trophic relationships. J. Anim. Ecol. 786-798.

Hussey, N.E., MacNeil, M.A., McMeans, B.C., Olin, J.A., Dudley, S.F., Cliff, G., Wintner, S.P., Fennessy, S.T., Fisk, A.T., 2014. Rescaling the trophic structure of marine food webs. Ecol. Lett. 17, 239-250.

IOTC, 2019. Report of the Seventh Session of the IOTC Working Party on Temperate Tunas (Data Preparatory Session). Kuala Lumpur, Malaysia, 14-17 January 2019, IOTC-2019-WPTmT07(DP)-R[E]: 43. Indian Ocean Tuna Commission, Seychelles.

Iverson, S.J., Field, C., Don Bowen, W., Blanchard, W., 2004. Quantitative fatty acid signature analysis: a new method of estimating predator diets. Ecol. Monogr. 74, 211-235.

Jennings, S., Maxwell, T.A., Schratzberger, M., Milligan, S.P., 2008. Body-size dependent temporal variations in nitrogen stable isotope ratios in food webs. Mar. Ecol. Prog. Ser. 370, 199-206.

Jennings, S., Warr, K.J., Mackinson, S., 2002. Use of size-based production and stable isotope analyses to predict trophic transfer efficiencies and predator-prey body mass ratios in food webs. Mar. Ecol. Prog. Ser. 240, $11-20$.

Jiang, H., Gao, K., 2004. Effects of lowering temperature during culture on the production of polyunsaturated fatty acids in the marine diatom Phaeodactylum tricornutum (bacillariophyceae) 1. J. Phycol. 40, 651-654.

Kerwath, S.E., Winker, H., West, W.M., 2012. Standardization of the catch per unit effort for albacore (Thunnus alalunga) for the South African tuna-pole (baitboat) fleet for the time series 1999-2010. ICCAT Collect. Vol. Sci. Pap. 68, 604-614.

Koz, A., 1995. A review of the trophic role of mesopelagic fish of the family Myctophidae in the Southern Ocean ecosystem. CCAMLR Sci. 2, 71-77.

Logan, J.M., Pethybridge, H., Lorrain, A., Somes, C., Allain, V., Bodin, N., Choy, C.A., Duffy, L., Goñi, N., Graham, B., Langlais, C., Menard, F., Olson, R., Young, J., in press. Global patterns and inferences of tuna movements and trophodynamics. Deep-Sea Res. II Top. Stud. Oceanogr.

Logan, J.M., Jardine, T.D., Miller, T.J., Bunn, S.E., Cunjak, R.A., Lutcavage, M.E., 2008. Lipid corrections in carbon and nitrogen stable isotope analyses: comparison of chemical extraction and modelling methods. J. Anim. Ecol. 77, 838-846.

Longhurst, A., 2006. Ecological geography of the sea. Academic Press, London.

Lorrain, A., Graham, B.S., Popp, B.N., Allain, V., Olson, R.J., Hunt, B.P., Potier, M., Fry, B., Galván-Magaña, F., Menkes, C.E., 2015. Nitrogen isotopic baselines and implications for estimating foraging habitat and 

Oceanogr. 113, 188-198.

Lorrain, A., Paulet, Y.-M., Chauvaud, L., Savoye, N., Donval, A., Saout, C., 2002. Differential $\delta^{13} \mathrm{C}$ and $\delta^{15} \mathrm{~N}$ signatures among scallop tissues: implications for ecology and physiology. J. Exp. Mar. Biol. Ecol. 275, $47-61$.

Lorrain, A., Pethybridge, H., Cassar, N., Receveur, A., Allain, V., Bodin, N., Bopp, L., Choy, A. C., Duffy, L., Fry, B., Goñi, N., Graham, B.S., Hobday, A.J., Logan, J.M., Ménard, F., Menkes, C., Olson, R.J., Point, D., Revill, A.T., Somes, C.J., Young, J.W., 2019. Trends in tuna carbon isotopes suggest global changes in pelagic phytoplankton communities. Glob Change Biol.1-13.

Lourey, M.J., Trull, T.W., Sigman, D.M., 2003. Sensitivity of $\delta^{15} \mathrm{~N}$ of nitrate, surface suspended and deep sinking particulate nitrogen to seasonal nitrate depletion in the Southern Ocean. Glob. Biogeochem. Cycles 17.

MacAvoy, S.E., Arneson, L.S., Bassett, E., 2006. Correlation of metabolism with tissue carbon and nitrogen turnover rate in small mammals. Oecologia 150, 190-201.

Madigan, D.J., Litvin, S.Y., Popp, B.N., Carlisle, A.B., Farwell, C.J., Block, B.A., 2012. Tissue turnover rates and isotopic trophic discrimination factors in the endothermic teleost, Pacific bluefin tuna (Thunnus orientalis). PLoS One 7, e49220.

Ménard, F., Benivary, H.D., Bodin, N., Coffineau, N., Le Loc’h, F., Mison, T., Richard, P., Potier, M., 2014. Stable isotope patterns in micronekton from the Mozambique Channel. Deep Sea Res. Part II Top. Stud. Oceanogr. 100, 153-163.

Ménard, F., Lorrain, A., Potier, M., Marsac, F., 2007. Isotopic evidence of distinct foraging ecology and movement pattern in two migratory predators (yellowfin tuna and swordfish) of the western Indian Ocean. Mar. Biol. 153, 141-152.

Möllmann, C., Diekmann, R., 2012. Marine ecosystem regime shifts induced by climate and overfishing: a review for the Northern Hemisphere. Adv. Ecol. Res. 47, 303-347.

Moore, C.M., Mills, M.M., Arrigo, K.R., Berman-Frank, I., Bopp, L., Boyd, P.W., Galbraith, E.D., Geider, R.J., Guieu, C., Jaccard, S.L., 2013. Processes and patterns of oceanic nutrient limitation. Nat. Geosci. 6, 701.

Mourente, G., Tocher, D.R., Sargent, J.R., 1991. Specific accumulation of docosahexaenoic acid (22: 6n- 3) in brain lipids during development of juvenile turbot Scophthalmus maximus L. Lipids 26, 871-877.

Mullin, M.M., Rau, G.H., Eppley, R.W., 1984. Stable nitrogen isotopes in zooplankton: some geographic and temporal variations in the North Pacific. Limnol. Oceanogr. 29, 1267-1273.

Navarro, J., Coll, M., Somes, C.J., Olson, R.J., 2013. Trophic niche of squids: Insights from isotopic data in marine systems worldwide. Deep Sea Res. Part II Top. Stud. Oceanogr. 95, 93-102.

Oksanen, J., Blanchet, F.G., Kindt, R., Legendre, P., Minchin, P.R., O’hara, R.B., Simpson, G.L., Solymos, P., Stevens, M.H.H., Wagner, H., 2018. Package 'vegan': Community Ecology Package. Version 2.

Olive, P.J., Pinnegar, J.K., Polunin, N.V., Richards, G., Welch, R., 2003. Isotope trophic-step fractionation: a dynamic equilibrium model. J. Anim. Ecol. 72, 608-617.

Oliver, M.J., Irwin, A.J., 2008. Objective global ocean biogeographic provinces. Geophys. Res. Lett. 35.

Olson, R.J., Popp, B.N., Graham, B.S., López-Ibarra, G.A., Galván-Magaña, F., Lennert-Cody, C.E., BocanegraCastillo, N., Wallsgrove, N.J., Gier, E., Alatorre-Ramírez, V., Ballance, L.T., Fry, B., 2010. Food-web inferences of stable isotope spatial patterns in copepods and yellowfin tuna in the pelagic eastern Pacific Ocean. Prog. Oceanogr. 86, 124-138.

Olsson, K., Nyström, P., Stenroth, P., Nilsson, E., Svensson, M., Granéli, W., 2008. The influence of food quality and availability on trophic position, carbon signature, and growth rate of an omnivorous crayfish. Can. J. Fish. Aquat. Sci. 65, 2293-2304.

Owens, N.J.P., Burkill, P.H., Mantoura, R.F.C., Woodward, E.M.S., Bellan, I.E., Aiken, J., Howland, R.J.M., Llewellyn, C.A., 1993. Size-fractionated primary production and nitrogen assimilation in the northwestern Indian Ocean. Deep Sea Res. Part II Top. Stud. Oceanogr. 40, 697-709.

Parrish, C.C., 2013. Lipids in marine ecosystems. ISRN Oceanogr. 2013.

Parrish, C.C., French, V.M., Whiticar, M.J., 2012. Lipid class and fatty acid composition of copepods (Calanus finmarchicus, C. glacialis, Pseudocalanus sp., Tisbe furcata and Nitokra lacustris) fed various combinations of autotrophic and heterotrophic protists. J. Plankton Res. 34, 356-375.

Parrish, C.C., Pethybridge, H., Young, J.W., Nichols, P.D., 2015. Spatial variation in fatty acid trophic markers in albacore tuna from the Southwestern Pacific Ocean—a potential ‘tropicalization'signal. Deep Sea Res. Part II Top. Stud. Oceanogr. 113, 199-207.

Parry, M., 2008. Trophic variation with length in two ommastrephid squids, Ommastrephes bartramii and Sthenoteuthis oualaniensis. Mar. Biol. 153, 249-256. 
Pethybridge, H., Choy, C.A., Logan, J.M., Allain, V., Lorrain, A., Bodin, N., Somes, C.J., Young, J., Ménard, F., Langlais, C., 2018. A global meta-analysis of marine predator nitrogen stable isotopes: Relationships between trophic structure and environmental conditions. Glob. Ecol. Biogeogr. 27, 1043-1055.

Pethybridge, H.R., Parrish, C.C., Morrongiello, J., Young, J.W., Farley, J.H., Gunasekera, R.M., Nichols, P.D., 2015a. Spatial patterns and temperature predictions of tuna fatty acids: tracing essential nutrients and changes in primary producers. PloS One 10, e0131598.

Pethybridge, H.R., Young, J.W., Kuhnert, P.M., Farley, J.H., 2015b. Using stable isotopes of albacore tuna and predictive models to characterize bioregions and examine ecological change in the SW Pacific Ocean. Prog. Oceanogr. 134, 293-303.

Pollard, R., Read, J., 2017. Circulation, stratification and seamounts in the Southwest Indian Ocean. Deep Sea Res. Part II Top. Stud. Oceanogr. 136, 36-43.

Polovina, J.J., Abecassis, M., Howell, E.A., Woodworth, P., 2009. Increases in the relative abundance of midtrophic level fishes concurrent with declines in apex predators in the subtropical North Pacific, 1996-2006. Fish. Bull. 107, 523-531.

Polovina, J.J., Howell, E., Kobayashi, D.R., Seki, M.P., 2001. The transition zone chlorophyll front, a dynamic global feature defining migration and forage habitat for marine resources. Prog. Oceanogr., Pacific climate variability and marine ecosystem impacts 49, 469-483.

Polovina, J.J., Woodworth, P.A., 2012. Declines in phytoplankton cell size in the subtropical oceans estimated from satellite remotely-sensed temperature and chlorophyll, 1998-2007. Deep Sea Res. Part II Top. Stud. Oceanogr. 77, 82-88.

Popp, B.N., Graham, B.S., Olson, R.J., Hannides, C.C., Lott, M.J., López-Ibarra, G.A., Galván-Magaña, F., Fry, B., 2007. Insight into the trophic ecology of yellowfin tuna, Thunnus albacares, from compound-specific nitrogen isotope analysis of proteinaceous amino acids. Terr. Ecol. 1, 173-190.

Post, D.M., 2002. Using stable isotopes to estimate trophic position: models, methods, and assumptions. Ecology 83, 703-718.

Potier, M., Marsac, F., Cherel, Y., Lucas, V., Sabatié, R., Maury, O., Ménard, F., 2007. Forage fauna in the diet of three large pelagic fishes (lancetfish, swordfish and yellowfin tuna) in the western equatorial Indian Ocean. Fish. Res. 83, 60-72.

Potier, M., Romanov, E., Cherel, Y., Sabatié, R., Zamorov, V., Ménard, F., 2008. Spatial distribution of Cubiceps pauciradiatus (Perciformes: Nomeidae) in the tropical Indian Ocean and its importance in the diet of large pelagic fishes. Aquat. Living Resour. 21, 123-134.

Quillfeldt, P., McGill, R.A., Furness, R.W., 2005. Diet and foraging areas of Southern Ocean seabirds and their prey inferred from stable isotopes: review and case study of Wilson's storm-petrel. Mar. Ecol. Prog. Ser. 295, 295-304.

Rainuzzo, J.R., Reitan, K.I., Olsen, Y., 1997. The significance of lipids at early stages of marine fish: a review. Aquaculture 155, 103-115.

Read, J.F., Lucas, M.I., Holley, S.E., Pollard, R.T., 2000. Phytoplankton, nutrients and hydrography in the frontal zone between the Southwest Indian Subtropical gyre and the Southern Ocean. Deep Sea Res. Part Oceanogr. Res. Pap. 47, 2341-2367.

Renaud, S.M., Zhou, H.C., Parry, D.L., Thinh, L.-V., Woo, K.C., 1995. Effect of temperature on the growth, total lipid content and fatty acid composition of recently isolated tropical microalgae Isochrysis sp., Nitzschia closterium, Nitzschia paleacea, and commercial species Isochrysis sp.(clone T. ISO). J. Appl. Phycol. 7, 595-602.

Revill, A.T., Young, J.W., Lansdell, M., 2009. Stable isotopic evidence for trophic groupings and bioregionalization of predators and their prey in oceanic waters off eastern Australia. Mar. Biol. 156, 12411253.

Reynolds, R.W., Smith, T.M., Liu, C., Chelton, D.B., Casey, K.S., Schlax, M.G., 2007. Daily high-resolutionblended analyses for sea surface temperature. J. Clim. 20, 5473-5496.

Robin, J.H., Regost, C., Arzel, J., Kaushik, S.J., 2003. Fatty acid profile of fish following a change in dietary fatty acid source: model of fatty acid composition with a dilution hypothesis. Aquaculture 225, 283-293.

Romanov, E., Nikolic, N., Dhurmeea, Z., Bodin, N., Puech, A., Norman, S., Hollanda, S., Bourjea, J., West, W., Potier, M., in press. Trophic ecology of albacore tuna Thunnus alalunga in the western tropical Indian Ocean and adjacent waters. Mar. Freshwater Res.

Romanov, E., Potier, M., Zamorov, V., Ménard, F., 2009. The swimming crab Charybdis smithii: distribution, biology and trophic role in the pelagic ecosystem of the western Indian Ocean. Mar. Biol. 156, 1089.

Roxy, M.K., Ritika, K., Terray, P., Masson, S., 2014. The Curious Case of Indian Ocean Warming. J. Clim. 27, 8501-8509. 
Sakshaug, E., 1977. Limiting nutrients and maximum growth rates for diatoms in Narragansett Bay. J. Exp. Mar. Biol. Ecol. 28, 109-123.

Sardenne, F., Bodin, N., Chassot, E., Amiel, A., Fouché, E., Degroote, M., Hollanda, S., Pethybridge, H., Lebreton, B., Guillou, G., Ménard, F., 2016. Trophic niches of sympatric tropical tuna in the Western Indian Ocean inferred by stable isotopes and neutral fatty acids. Prog. Oceanogr. 146, 75-88.

Sardenne, F., Ménard, F., Degroote, M., Fouché, E., Guillou, G., Lebreton, B., Hollanda, S.J., Bodin, N., 2015. Methods of lipid-normalization for multi-tissue stable isotope analyses in tropical tuna. Rapid Commun. Mass Spectrom. 29, 1253-1267.

Scharf, F.S., Juanes, F., Rountree, R.A., 2000. Predator size-prey size relationships of marine fish predators: interspecific variation and effects of ontogeny and body size on trophic-niche breadth. Mar. Ecol. Prog. Ser. 208, 229-248.

Schott, F.A., Dengler, M., Schoenefeldt, R., 2002. The shallow overturning circulation of the Indian Ocean. Prog. Oceanogr. 53, 57-103.

Schott, F.A., McCreary Jr., J.P., 2001. The monsoon circulation of the Indian Ocean. Prog. Oceanogr. 51, 1-123.

Sinclair, M., Arnason, R., Csirke, J., Karnicki, Z., Sigurjonsson, J., Rune Skjoldal, H., Valdimarsson, G., 2002. Responsible fisheries in the marine ecosystem. Fish. Res. 58, 255-265.

Smayda, T.J., 2002. Adaptive ecology, growth strategies and the global bloom expansion of dinoflagellates. J. Oceanogr. 58, 281-294.

Smith, R.J., Hobson, K.A., Koopman, H.N., Lavigne, D.M., 1996. Distinguishing between populations of freshand salt-water harbour seals (Phoca vitulina) using stable-isotope ratios and fatty acid profiles. Can. J. Fish. Aquat. Sci. 53, 272-279.

Somes, C.J., Oschlies, A., 2015. On the influence of "non-Redfield” dissolved organic nutrient dynamics on the spatial distribution of N2 fixation and the size of the marine fixed nitrogen inventory. Glob. Biogeochem. Cycles, 29, 973-993.

Somes, C.J., Schmittner, A., Galbraith, E.D., Lehmann, M.F., Altabet, M.A., Montoya, J.P., Letelier, R.M., Mix, A.C., Bourbonnais, A., Eby, M., 2010. Simulating the global distribution of nitrogen isotopes in the ocean. Glob. Biogeochem. Cycles 24.

Somes, C.J., Schmittner, A., Muglia, J., Oschlies, A., 2017. A three-dimensional model of the marine nitrogen cycle during the last glacial maximum constrained by sedimentary isotopes. Front. Mar. Sci. 4, 108.

Sonnekus, M.J., Bornman, T.G., Campbell, E.E., 2017. Phytoplankton and nutrient dynamics of six South West Indian Ocean seamounts. Deep Sea Res. Part II Top. Stud. Oceanogr. 136, 59-72.

Suda, A., 1974. Recent Status of resources of tuna exploited by longline fishery in the Indian Ocean. Far Seas Fish. Res. Lab. Bull. 10, 27-62.

Suikkanen, S., Pulina, S., Engström-Öst, J., Lehtiniemi, M., Lehtinen, S., Brutemark, A., 2013. Climate change and eutrophication induced shifts in northern summer plankton communities. PLoS One 8, e66475.

Thompson, P.A., Guo, M., Harrison, P.J., 1992. Effects of variation in temperature. 1. On the biochemical composition of eight species of marine phytoplankton. J. Phycol. 28, 481-488.

Tocher, D.R., Sargent, J.R., 1990. Effect of temperature on the incorporation into phospholipid classes and metabolismvia desaturation and elongation of $n-3$ and n- 6 polyunsaturated fatty acids in fish cells in culture. Lipids 25, 435-442.

Tomczak, M., Godfrey, J.S., 2003. Regional oceanography: an introduction, Second. ed. Daya Publishing House, Delhi.

Trull, T.W., Armand, L., 2001. Insights into Southern Ocean carbon export from the $\delta^{13} \mathrm{C}$ of particles and dissolved inorganic carbon during the SOIREE iron release experiment. Deep Sea Res. Part II Top. Stud. Oceanogr. 48, 2655-2680.

Underwood, A.J., 1997. Experiments in ecology: their logical design and interpretation using analysis of variance. Cambridge University Press.

Vander Zanden, M.J., Fetzer, W.W., 2007. Global patterns of aquatic food chain length. Oikos 116, $1378-1388$.

Vander Zanden, M.J., Rasmussen, J.B., 2001. Variation in $\delta^{15} \mathrm{~N}$ and $\delta^{13} \mathrm{C}$ trophic fractionation: implications for aquatic food web studies. Limnol. Oceanogr. 46, 2061-2066.

Vander Zanden, M.J., Rasmussen, J.B., 1999. Primary consumer $\delta^{13} \mathrm{C}$ and $\delta^{15} \mathrm{~N}$ and the trophic position of aquatic consumers. Ecology 80, 1395-1404.

Vanderklift, M.A., Ponsard, S., 2003. Sources of variation in consumer-diet $\delta^{15} \mathrm{~N}$ enrichment: a meta-analysis. Oecologia 136, 169-182.

Vecsei, A., 2003. Nutrient control of the global occurrence of isolated carbonate banks. Int. J. Earth Sci. 92, 476481. 
Veldhuis, M.J.W., Kraay, G.W., Van Bleijswijk, J.D.L., Baars, M.A., 1997. Seasonal and spatial variability in phytoplankton biomass, productivity and growth in the northwestern Indian Ocean: the southwest and northeast monsoon, 1992-1993. Deep Sea Res. Part Oceanogr. Res. Pap. 44, 425-449.

Vipin, P.M., Ravi, R., Fernandez, T.J., Pradeep, K., Boopendranath, M.R., Remesan, M.P., 2012. Distribution of myctophid resources in the Indian Ocean. Rev. Fish Biol. Fish. 22, 423-436.

Watanabe, T., 1982. Lipid nutrition in fish. Comp. Biochem. Physiol. Part B Comp. Biochem. 73, 3-15.

Wilkerson, F.P., Dugdale, R.C., 1987. The use of large shipboard barrels and drifters to study the effects of coastal upwelling on phytoplankton dynamics1, 2. Limnol. Oceanogr. 32, 368-382.

Williams, A.J., Allain, V., Nicol, S.J., Evans, K.J., Hoyle, S.D., Dupoux, C., Vourey, E., Dubosc, J., 2015. Vertical behavior and diet of albacore tuna (Thunnus alalunga) vary with latitude in the South Pacific Ocean. Deep Sea Res. Part II Top. Stud. Oceanogr. 113, 154-169.

Wood, S., 2006. Generalized additive models: an introduction with R. Chapman and Hall/CRC, Boca Raton, FL.

Wood, S., Scheipl, F., 2017. Package "gamm4": Generalized Additive Mixed Models using mgcv and lme4. Package version 0.2-5.

Young, J., Lansdell, M., Riddoch, S., Revill, A., 2006. Feeding ecology of broadbill swordfish, Xiphias gladius, off eastern Australia in relation to physical and environmental variables. Bull. Mar. Sci. 79, 793-809.

Young, J.W., Bradford, R., Lamb, T.D., Clementson, L.A., Kloser, R., Galea, H., 2001. Yellowfin tuna (Thunnus albacares) aggregations along the shelf break off south-eastern Australia: links between inshore and offshore processes. Mar. Freshw. Res. 52, 463-474.

Young, J.W., Lansdell, M.J., Campbell, R.A., Cooper, S.P., Juanes, F., Guest, M.A., 2010. Feeding ecology and niche segregation in oceanic top predators off eastern Australia. Mar. Biol. 157, 2347-2368.

Young, J.W., Olson, R.J., Ménard, F., Kuhnert, P.M., Duffy, L.M., Allain, V., Logan, J.M., Lorrain, A., Somes, C.J., Graham, B., 2015. Setting the stage for a global-scale trophic analysis of marine top predators: a multiworkshop review. Rev. Fish Biol. Fish. 25, 261-272.

Zimmerman, R.C., Kremer, J.N., Dugdale, R.C., 1987. Acceleration of nutrient uptake by phytoplankton in a coastal upwelling ecosystem: A modeling analysis1, 1. Limnol. Oceanogr. 32, 359-367.

Zudaire, I., Murua, H., Grande, M., Goñi, N., Potier, M., Ménard, F., Chassot, E., Bodin, N., 2015. Variations in the diet and stable isotope ratios during the ovarian development of female yellowfin tuna (Thunnus albacares) in the Western Indian Ocean. Mar. Biol. 162, 2363-2377. 


\section{HIGHLIGHTS}

- A multi-tracer approach was taken to examine the trophodynamics of albacore tuna

- Albacore inhabiting temperate southern waters feed on a diatom-based food web

- Albacore trophic position was lowest in the northern tropical waters

- Ontogenetic shifts in stable carbon and nitrogen isotopes were observed

- High seawater temperature lowers the nutritional condition of albacore 


\section{AUTHOR DECLARATION}

We wish to confirm that there are no known conflicts of interest associated with this publication and there has been no financial support for this work that could have influenced its outcome.

We confirm that the manuscript has been read and approved by all named authors and that there are no other persons who satisfied the criteria for authorship but are not listed. We further confirm that the order of authors listed in the manuscript has been approved by all of us.

We confirm that we have given due consideration to the protection of intellectual property associated with this work and that there are no impediments to publication, including the timing of publication, with respect to intellectual property. In so doing we confirm that we have followed the regulations of our institutions concerning intellectual property.

We understand that the Corresponding Author is the sole contact for the Editorial process (including Editorial Manager and direct communications with the office). She is responsible for communicating with the other authors about progress, submissions of revisions and final approval of proofs. We confirm that we have provided a current, correct email address which is accessible by the Corresponding Author and which has been configured to accept email from dzahirah@hotmail.com.

Signed by all authors as follows:

Zahirah Dhurmeea

Heidi Pethybridge

Clothilde Langlais

Christopher J. Somes

Natacha Nikolic

Jérôme Bourjea

Chandani Appadoo

Nathalie Bodin 